\title{
Mesoscale finite element analysis of cracked composite laminates under out-of-plane loads using 3D periodic boundary conditions.
}

\author{
D. Garoz ${ }^{\mathrm{a}, \mathrm{b},{ }^{*}}$, M. Hajikazemi ${ }^{\mathrm{a}, \mathrm{c}}$, T.D. Dinh ${ }^{\mathrm{a}, \mathrm{b}}$, W. Van Paepegem ${ }^{\mathrm{a}}$
}

${ }^{a}$ Ghent University, Department of Materials, Textiles and Chemical Engineering - MaTCh, Tech Lane Ghent Science Park - Campus A, Technologiepark Zwijnaarde 46, 9052 Zwijnaarde (Gent), Belgium. ${ }^{b}$ SIM vzw, Technologiepark 48, 9052 Zwijnaarde (Gent), Belgium.

${ }^{c}$ Dutch Polymer Institute (DPI), P.O. Box 902, 5600 AX, Eindhoven, The Netherlands

*Corresponding author: david.garoz@ugent.be

\begin{abstract}
The behavior of composite materials under out-of-plane loads is strongly affected by the presence of transverse ply cracks. The cracks perturb the distribution of stresses leading to large out-of-plane shear stiffness reductions. It is crucial to include these effects in the damage material models to improve their accuracy. Therefore, the stress transfer and stiffness reduction in cracked laminates have been studied with a mesoscale finite element model (FEM) under general in-plane, out-of-plane normal and shear loads. A symmetric laminate containing ply cracks in a single orientation has been considered under the hypothesis of periodicity using a novel relaxed three-dimensional formulation of Periodic Boundary Conditions (PBCs). The local stresses have been verified versus different analytical and numerical methods. In addition, the degraded effective thermo-elastic constants involving out-of-plane properties have been calculated as a function of crack density. Both uniform and non-uniform distributions of cracks have been considered for different lay-ups including angleply and unbalanced laminates. The effect of contact between the crack surfaces has been studied for specific loading conditions. It is shown that a single formulation based on three-dimensional periodic boundary conditions is sufficient to determine the interfacial stresses and the complete thermo-elastic constants under in-plane and out-of-plane loads accurately.
\end{abstract}

Key words: Cracked laminate; Transverse ply cracks; Out-of-plane load; Finite Elements; Periodic Boundary Conditions

\section{Introduction}

30 Accurate damage models of fiber-reinforced composite materials must be developed to design better composite structures avoiding too many expensive and time-consuming experiments. The complex damage behavior of composite laminates is generally described by four damage modes: debonding between fiber and matrix, transverse ply cracking, delamination at the interface between the plies and fiber fracture [1,2]. The evolution of damage depends on the laminate architecture and loading conditions. Moreover, the four damage modes are coupled and can trigger or suppress each other while the composite structure is loaded [3,4]. However, ply cracking is typically the first ply level damage mode which can lead to large stiffness reductions and trigger other damage modes. 
When ply cracks appear in a laminate, both the in-plane and out-of-plane material properties of the laminate degrade [5-8]. Also, the large stress gradients at the tips of ply cracks can initiate more detrimental damage modes like delamination and fiber breakage $[6,9,10]$. There are many applications of laminates under in-plane loads, however, out-of-plane normal and shear loads can also be found for example within bolted joints and pressure vessels. Also, out-of-plane deformations can be found near the free edges of a laminate. Therefore, it is important to evaluate the stress transfer in cracked laminates under both the in-plane and out-of-plane normal and shear loads.

The local stresses can be evaluated using a multiscale approach for which the cracked laminate is described with a Representative Unit Cell (RUC) with a specific crack density. Different analytical and numerical methods have been developed to report the local stresses in a laminate under in-plane loads [4,11-16] or flexural loads, bending and torsion [6,17-21]. Analytically, the local stresses in a cracked laminate under out-of-plane normal loads can be predicted [11]. However, the stress transfer due to out-of-plane shear load has only been studied recently using a Variational Approach (VA) [8]. Although the local stresses in a cracked laminate under out-of-plane loads have been less studied in the literature, they have quite important effects on the stiffness reduction and damage evolution.

The present paper describes novel results about the local stresses in a cracked laminate under general out-of-plane and in-plane loads using a Finite Element Model (FEM) of the RUC while the effects of thermally induced residual stresses are taken into account. The out-of-plane shear loads have been included using a novel relaxed implementation of the three-dimensional (3D) formulation of Periodic Boundary Conditions (PBCs) where the relative displacement normal to the top and bottom surfaces is released. The relaxed 3D PBCs have been verified comparing the local stresses under in-plane loads with the results obtained from different analytical and numerical methods [17,22,23]. Then, new results of local stresses under out-of-plane load have been reported.

In addition, the degradation of all relevant thermo-elastic constants is considered including the normal and shear out of plane properties. The effects of contact between the crack surfaces on the local stresses and thermo-elastic constants have been studied.

Finally, the effect of the non-uniform distribution of cracks has been studied. Novel results of local stresses and stiffness reductions under out-of-plane loads have been reported for a symmetric laminate containing non-uniformly distributed ply cracks. The results show that the new formulation of relaxed 3D PBCs is the best approach to account for the out-of-plane degraded properties, without modifying the results for the in-plane properties.

\section{Finite Element Model}

70 An arbitrary symmetric multilayered laminate has been modeled using Finite Elements (FE). A rectangular Cartesian coordinate is located at the center of the laminate. The $x$-axis specifies the inplane axial direction while the $y$-axis defines the in-plane transverse direction and $z$-axis defines the through-thickness direction, see Figure 1. The considered laminate geometries, material properties and boundary conditions are described in the next sections. 


\subsection{Geometry and Mesh}

A Representative Unit Cell (RUC) is used to describe the full laminate with a certain number of plies. Each ply is a part of the geometry which has the ply properties oriented with its local coordinate system. They are numbered from bottom to top and symmetry about the middle plane is preserved. The dimensions of the RUC are $l_{x}$ in the axial direction, $l_{y}$ along the in-plane transverse direction and $l_{z}$ through the thickness.

Cracks are introduced at the $90^{\circ}$ plies keeping the symmetry about the middle plane. This is not a limitation because general loading conditions are considered and if cracks appear in another orientation, the laminate and the applied stresses can rotate so that the crack planes are again parallel to the fibers in a $90^{\circ}$ ply. The vertical cracks which run through the thickness of the ply are located at the middle of the axial length $l_{x}$. Therefore, $l_{x}$ is defined as the inverse of the crack density, $\rho=1 / l_{x}$.

The thickness of the plies is constant $t_{p l y}$, and then the laminate thickness $l_{z}$ is calculated multiplying the number of plies by their thickness. In the coordinate system where cracks are in $90^{\circ}$ plies, the depth of the laminate $l_{y}$ does not play any role, and its effect is evaluated with a parametric study.

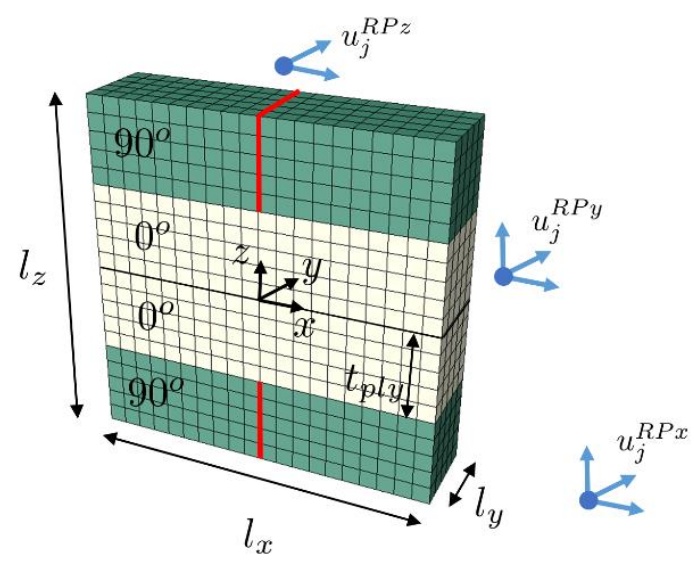

Figure 1. Finite element geometry of the RUC for a symmetric cracked laminate, [90/0]s. Cracks are located in the $90^{\circ}$ plies at the middle of the length of the RUC. The three control points are represented with their active components in the formulation of relaxed $3 D$ PBCs.

The geometry is discretized with hexahedral finite elements to build a conformal mesh. The 8-node elements with full integration have been used in the simulations (C3D8 in Abaqus notation [24]). The cracks have been introduced in the model by duplicating the nodes at the crack surface except for the nodes at the crack tip. Therefore, new elements are created on both sides of the crack plane. They are called seam cracks. This approach allows to include contact between the crack surfaces to avoid interpenetration. For each simulation, it is mentioned whenever the contact algorithm is activated or not.

\subsection{Material}

Uniform homogenized ply properties in the linear elastic regime are used in this study. This simplification dismisses the microstructure of the unidirectional plies, where the random distribution of fibers introduces local effects such as stress concentrations. Therefore, the results must be 
understood in the framework of a mesoscale laminate model with homogenized linear elastic ply properties.

Six linear elastic material models have been used to represent the general behavior of glass and carbon fiber reinforced plies. Table 1 shows the transversely isotropic material properties of the ply for each material system and the ply thickness.

Table 1. Elastic properties of each ply. CFRP = Carbon fiber reinforced polymer; GFRP = Glass fiber reinforced polymer; $E_{11}=$ In-plane axial modulus; $E_{22}=$ In-plane transverse modulus; $E_{33}=$ Out-of-plane normal modulus; $v_{12}$ and $v_{13}=I n-p l a n e ~ P o i s s o n ' s$ ratio; $v_{23}=$ Transverse Poisson's ratio; $G_{12}$ and $G_{13}=I n$ plane shear elastic modulus; $G_{23}=E_{22} /\left(1+v_{23}\right)$ Out-of-plane shear elastic modulus; $\alpha_{11}=$ Longitudinal coefficient of thermal expansion; $\alpha_{22}=$ Transverse coefficient of thermal expansion.

\begin{tabular}{cccccccccc}
\hline Material & Sets & $\begin{array}{c}E_{11} \\
(\mathrm{GPa})\end{array}$ & $\begin{array}{c}E_{22}=E_{33} \\
(\mathrm{GPa})\end{array}$ & $\begin{array}{c}v_{12}=v_{13} \\
(-)\end{array}$ & $\begin{array}{c}v_{23} \\
(-)\end{array}$ & $\begin{array}{c}G_{12}=G_{13} \\
(\mathrm{GPa})\end{array}$ & $\begin{array}{c}\alpha_{11} \\
10^{-6}(1 / \mathrm{K})\end{array}$ & $\begin{array}{c}\alpha_{22}=\alpha_{33} \\
10^{-6}(1 / \mathrm{K})\end{array}$ & $\begin{array}{c}t_{p l y} \\
(\mathrm{~mm})\end{array}$ \\
\hline CFRP1 [25] & Set-1 & 134 & 9.8 & 0.3 & 0.361 & 5.5 & -0.9 & 28.8 & 0.144 \\
GFRP1 [26] & Set-2 & 44.7 & 12.7 & 0.297 & 0.411 & 5.8 & 8.43 & 18.44 & 0.144 \\
GFRP2 [22] & Set-3 & 46.0 & 13.0 & 0.3 & 0.42 & 5.0 & - & - & 0.5 \\
GFRP3 [27] & Set-4 & 41.7 & 13.0 & 0.3 & 0.42 & 3.4 & 6.72 & 29.3 & 0.203 \\
GFRP4 [17] & Set-5 & 45.6 & 16.2 & 0.278 & 0.4 & 5.83 & 8.6 & 26.4 & 0.127 \\
GFRP5 [14] & Set-6 & 43 & 13 & 0.3 & 0.42 & 3.4 & - & - & 0.61 \\
\hline
\end{tabular}

\subsection{Boundary conditions}

$1151^{\text {st }}$ order Periodic Boundary Conditions (PBC) have been implemented in the three directions of the global coordinate system (3D PBCs). Relative displacements between counterpart nodes located on opposite surfaces of the RUC are described by,

$$
u_{j}^{i+}+u_{j}^{i-}=\varepsilon_{i j} l_{j} \text { with } i, j=x, y, z
$$

where $\varepsilon_{i j}$ is the macro-strain applied on the laminate and $l_{j}$ is the length of the RUC in $j$ direction. The superscripts $i+$ and $i$ - indicate the opposite surfaces perpendicular to the $i$-direction.

120 The implementation of PBCs avoids the redundant constraints between the nodes located at the edges of the RUC. First, the kinematic equations (1) are written between all the nodes in the surface $y$ - and their counterpart nodes in $y+$. Then, the equations between the nodes in the surface $x$ - and their counterpart nodes in $x+$ are established excluding the nodes at the edges where both surfaces $(x-, x+)$ join the surface $y+$. Finally, the equations between the nodes in the surface $z$ - and their counterpart nodes in $z+$ are written excluding all the nodes at the edges of both surfaces. More detail about the equations of the implementation is reported in Ref. [28]. It is worth mentioning that this implementation of PBCs distinguishes the overlap nodes introduced by the discrete cracks. Therefore, each overlap node on one surface is related to its counterpart node on the opposite surface.

The load conditions are introduced through the displacements of three control nodes, $u_{j}^{R P i}$ with $i, j=$ $x, y, z$, see Figure 1 . The macro-strains of the laminate are related to these displacements as follows:

$$
\varepsilon_{i j} l_{j}=u_{j}^{R P i} \text { with } i, j=x, y, z
$$

The three control nodes do not belong to the mesh, but they are included in the model via kinematic equations. Because they are points of the model, displacements or concentrated loads can be applied to each component. If no displacement is applied in one component, the solver interprets that the 
concentrated force is zero in this component and it adds the necessary equation of equilibrium. Initially, the formulation allows to introduce the full macro-strain tensor with 9 independent components as input. To fulfill the equilibrium of the RUC, three kinematic constraints link the transverse displacements of control nodes keeping the symmetry of the shear macro-strains, $\varepsilon_{i j}=\varepsilon_{j i}$.

$$
\frac{u_{j}^{R P i}}{l_{j}}=\frac{u_{i}^{R P j}}{l_{i}} \text { with } i, j=x, y, z
$$

The 3D PBCs have been relaxed by releasing the constraint in the normal direction between the nodes at top and bottom surfaces. Therefore, the equations (1) between the top and bottom surfaces $(i=z)$ are only applied in the shear components $(j=x, y)$.

The relaxed 3D PBCs can introduce the in-plane normal and shear loads, as well as the out-of-plane shear load conditions. A uniform normal stress has been applied on the top and bottom surfaces to study the out-of-plane normal behavior.

Finally, a thermal load can be included using an initial and final predefined uniform field of temperature in degrees Celsius.

\subsection{Homogenization}

After the analysis of the RUC with the imposed macro-strains, the average stresses $\tilde{\sigma}_{i j}$ must be computed. Usually, the average stresses can be computed evaluating the volume integral of local stresses $\sigma_{i j}$, but it is computationally efficient to simplify it using the kinematic PBC between macrostrains and concentrated forces in the control nodes [29]. Therefore, the external forces at the control nodes, $f_{j}^{R P i}$, are the only ones to contribute to the external power. Considering the Hill-Mandel principle, the external power of the RUC (meso-scale) must be equal to the internal power of the macro-scale. Then, the average or resultant forces can be computed from the external forces on the control nodes. This approach has been used by several authors for a RUC containing continuous or discrete damage, such as cracks $[6,30,31]$.

The average stresses can be noted as

$$
\tilde{\sigma}_{x x}=\frac{f_{x}^{R P x}}{l_{y} l_{z}} ; \tilde{\sigma}_{x y}=\frac{f_{y}^{R P x}}{l_{y} l_{z}} ; \tilde{\sigma}_{x z}=\frac{f_{z}^{R P x}}{l_{y} l_{z}} ; \tilde{\sigma}_{y y}=\frac{f_{y}^{R P y}}{l_{x} l_{z}} ; \tilde{\sigma}_{y z}=\frac{f_{z}^{R P y}}{l_{x} l_{z}} ; \tilde{\sigma}_{z z}=\frac{f_{z}^{R P z}}{l_{x} l_{y}}
$$

Note that there are no concentrated forces which contribute to the symmetric shear stresses $\tilde{\sigma}_{y x}, \tilde{\sigma}_{z x}$ or $\tilde{\sigma}_{z y}$ because the shear macro-strains are kinematically restricted to fulfil the equilibrium of the RUC. Using 3D PBCs, any load or combination can be introduced as concentrated forces on the control nodes. Then, the average strains are obtained from the displacements of the control nodes using equation (2).

In the case of relaxed 3D PBCs, the normal stress along the $z$-direction cannot be introduced as a concentrated force. As mentioned before, when using relaxed 3D PBCs, the out-of-plane normal load is introduced with uniform stresses on the top and bottom surfaces. The Hill-Mandel condition is still valid under uniform traction boundary conditions [29,32]; therefore, the out-of-plane normal relative 
displacement $u_{z}^{R P z}$ is evaluated as the average of the relative displacements between the counterpart nodes at the top and bottom surfaces. Then, the average strain is calculated as $\varepsilon_{z z}=u_{z}^{R P z} / l_{z}$.

The cross-section forces are described as the average stresses multiplied by the thickness of the laminate, $N_{i j}=\tilde{\sigma}_{i j} l_{z}$.

In this study, the thermo-elastic constants have been calculated imposing six loading states, three axial and three shear loads. Each state has a unique nonzero component, and then the compliance matrix coefficients are obtained from the average retrieved strains when there is no increment of temperature, $\Delta T$.

$$
\left[\begin{array}{c}
\varepsilon_{x x} \\
\varepsilon_{y y} \\
\varepsilon_{z z} \\
2 \varepsilon_{y z} \\
2 \varepsilon_{x z} \\
2 \varepsilon_{x y}
\end{array}\right]=\left[\begin{array}{llllll}
S_{11} & S_{12} & S_{13} & S_{14} & S_{15} & S_{16} \\
S_{21} & S_{22} & S_{23} & S_{24} & S_{25} & S_{26} \\
S_{31} & S_{32} & S_{33} & S_{34} & S_{35} & S_{36} \\
S_{41} & S_{42} & S_{43} & S_{44} & S_{45} & S_{46} \\
S_{51} & S_{52} & S_{53} & S_{54} & S_{55} & S_{56} \\
S_{61} & S_{62} & S_{63} & S_{64} & S_{65} & S_{66}
\end{array}\right]\left[\begin{array}{c}
\tilde{\sigma}_{x x} \\
\tilde{\sigma}_{y y} \\
\tilde{\sigma}_{z z} \\
\tilde{\sigma}_{y z} \\
\tilde{\sigma}_{x z} \\
\tilde{\sigma}_{x y}
\end{array}\right]+\left[\begin{array}{c}
\alpha_{x x} \\
\alpha_{y y} \\
\alpha_{z z} \\
\alpha_{y z} \\
\alpha_{x z} \\
\alpha_{x y}
\end{array}\right] \Delta T
$$

The coefficients of thermal expansion (TEC) are obtained with one load state more, where all the concentrated forces are zero while a $\Delta T$ is applied.

Although no initial hypotheses have been made about the coefficients of the compliance matrix, it is expected to obtain a symmetric matrix, $S_{i j}=S_{j i}$. This condition is checked in the numerical software and turned out to be true for all assumed input values.

The effective engineering properties are defined as

$$
\begin{aligned}
& E_{A}=1 / S_{11}, E_{T}=1 / S_{22}, E_{t}=1 / S_{33}, G_{A}=1 / S_{66}, G_{t}=1 / S_{44}, G_{a}=1 / S_{55}, \\
& v_{A}=-S_{12} / S_{11}, v_{t}=-S_{23} / S_{22}, v_{a}=-S_{13} / S_{11}, \\
& \lambda_{x}=-S_{16} / S_{11}, \lambda_{y}=-S_{26} / S_{11}, \lambda_{z}=-S_{36} / S_{11}, \lambda_{S}=-S_{45} / S_{55}, \\
& \alpha_{A}=\alpha_{x x}, \alpha_{T}=\alpha_{y y}, \alpha_{t}=\alpha_{z z}, \alpha_{S}=\alpha_{x y}
\end{aligned}
$$

$E_{A}, E_{T}$ and $E_{t}$ are, respectively, the axial, transverse and through-thickness Young's modulus of the laminate. $G_{A}, G_{t}$ and $G_{a}$ are, respectively, the in-plane, transverse out-of-plane and axial out-of-plane shear modulus of the laminate. Similarly, $v_{A}, v_{t}$ and $v_{a}$ are the Poisson's ratios. $\lambda_{x}, \lambda_{y}$ and $\lambda_{z}$ are the coupling coefficients between normal and in-plane shear load along the three directions, while $\lambda_{S}$ is the coupling coefficient between out-of-plane shear loads. $\alpha_{A}, \alpha_{T}, \alpha_{t}$ and $\alpha_{S}$ are the in-plane axial, transverse, through-thickness and shear thermal expansion coefficients (TEC), respectively.

It is noteworthy that the homogenized coefficients have been calculated without considering contact between the crack surfaces. It is assumed that the cracks are always open. Nevertheless, the difference between the stress fields with and without contacts have been studied for specific load cases in section 3.1.5. 


\section{Results for laminates with uniform distribution of cracks}

In this section, the local stress fields and stiffness reduction in symmetric cracked laminates are studied. Firstly, the local stresses along different planes are described. Results with the in-plane loads are compared with those obtained from other methods, and the local stresses due to out-of-plane loads are reported. In addition, the effect of contact between the crack surfaces has been studied for specific cases. Then, stiffness reduction is calculated as a function of the crack density for different laminates. The predicted in-plane laminate thermo-elastic constants have been compared with experiments and other analytical and numerical methods. Finally, the reduction of out-of-plane shear stiffness is reported for different laminates including angle-ply laminates.

\subsection{Local stress analysis}

Figure 2 shows the deformation and local stress field for the six loading states and the thermal load on the [90/0]s laminate made of GFRP1 (Set-2) and crack density of 2/mm. The relaxed 3D PBC have been used and the contact between crack surfaces has not been considered. The normal and shear average stresses have been set to $1 \mathrm{MPa}, \tilde{\sigma}_{i j}=1 \mathrm{MPa}\left(N_{i j}=0.576 \mathrm{~N} / \mathrm{mm}\right)$. The normal loads, $\tilde{\sigma}_{x x}$, $\tilde{\sigma}_{y y}$ and $\tilde{\sigma}_{z z}$, open the crack surfaces and create a stress singularity at the crack tips. Also, the in-plane shear load $\tilde{\sigma}_{x y}$ and the out-of-plane shear load $\tilde{\sigma}_{x z}$ slide the crack surfaces leading to stress singularities located at the crack tips. For balanced laminates with cracks in the $90^{\circ}$ plies, the shear load $\tilde{\sigma}_{y z}$ does not open the crack surfaces and the results are the same as an intact laminate with constant shear stresses. Finally, the temperature difference $\Delta T=-1 \mathrm{~K}$ opens the cracks and introduces stress singularities at the crack tips. In all the load cases, the stress fields are constant along the $y$-direction and the stresses are continuous between opposite faces in $x$ - and $z$-directions.
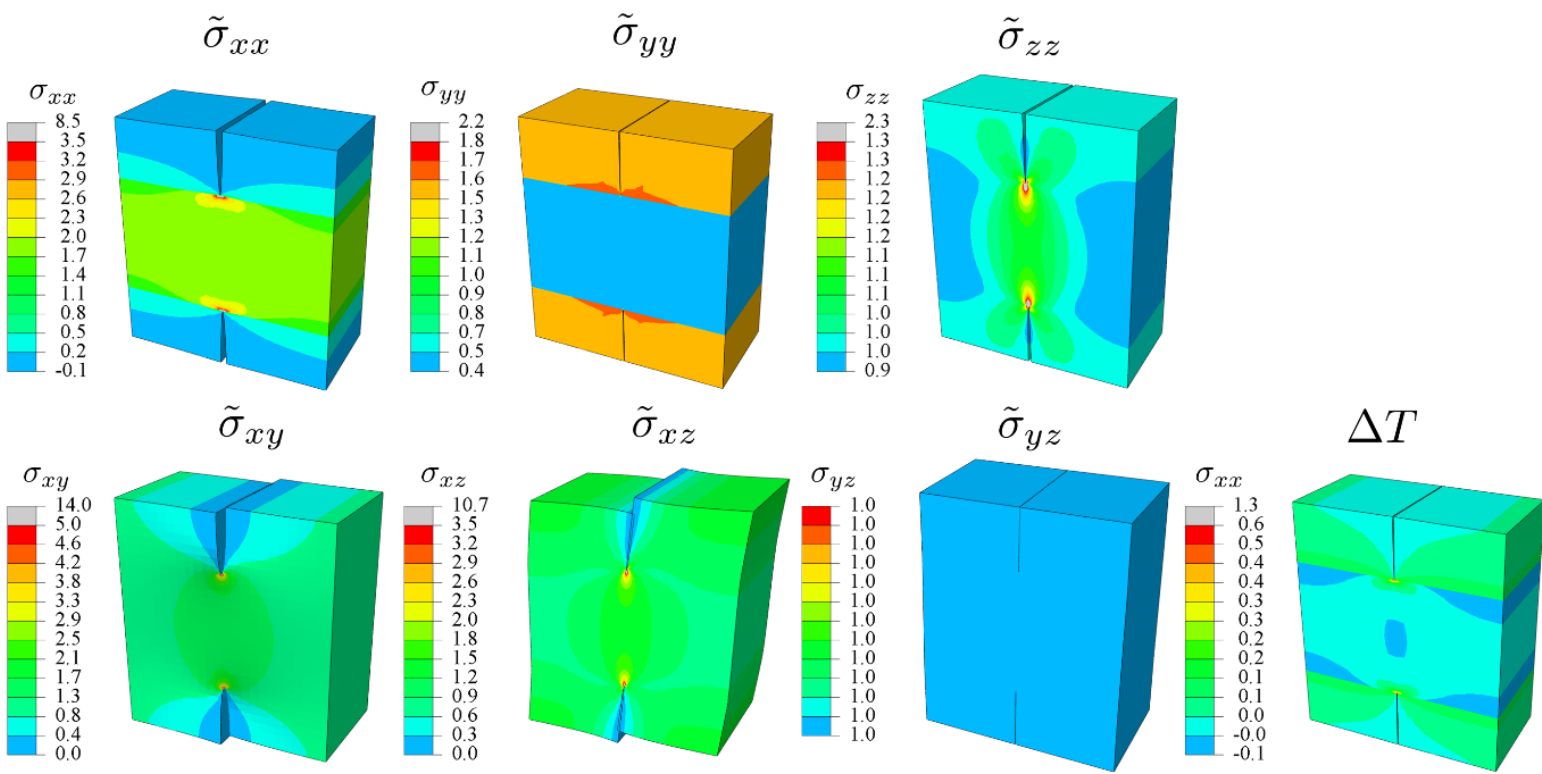

Figure 2. Deformation modes and local stresses of the 6 load cases under normalized average stress $\widetilde{\sigma}_{\mathrm{ij}}=1$

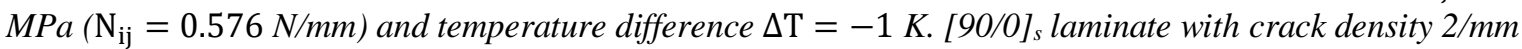
made of GFRP1 (Set-2). The deformation has a scale factor of 750, stress units are in MPa. 
It is noted that for a displacement-based FE formulation, the stress is usually described inside each element with one or several integration points. Therefore, the stress values at singularities (crack tip) or free surfaces depend on the element size. A convergence study has been performed to describe the singularity at the crack tip and the traction free condition on the free surfaces with high accuracy.

\subsubsection{Mesh convergence of relaxed 3D PBC}

A mesh convergence study has been done for a cross-ply laminate [90/0]s made of CFRP1 (Set-1) with a crack density of $2 / \mathrm{mm}$. A structured mesh with full integration elements has been used. First, the convergence of a uniform mesh has been checked decreasing the size of all elements. Then, a mesh refinement has been performed at the elements around the crack tip and next to the free surfaces

225 (top and bottom) keeping a uniform mesh around. The size of the elements has been controlled using a global seed parameter and a refined element size.

Figure 3 (a) shows the axial distribution of shear stress $\sigma_{x z}$ along the interface 90/0 from the crack tip. Out-of-plane average stress $\tilde{\sigma}_{x z}=1 \mathrm{MPa}\left(N_{x z}=0.576 \mathrm{~N} / \mathrm{mm}\right)$ has been used as a loading condition using relaxed 3D PBCs. The stress singularity is better described when the element size of the mesh decreases. Results with a uniform mesh decreasing the global element size from 0.03 to $0.005 \mathrm{~mm}$ are represented in Figure 3 (a) (Uniform seed). Above these results, the convergence study of a mesh with a fixed global seed of $0.01 \mathrm{~mm}$ and refined element from 0.005 to $0.0002 \mathrm{~mm}$ has been added (Refined seed). It has been found that the stress behavior of the uniform mesh and the refined one is the same when the size of the elements around the crack tip is equal for both meshes. The maximum stress difference close to the crack tip is lower than $6 \%$ when the uniform mesh with a global seed of $0.005 \mathrm{~mm}$ compares with the refined mesh with global seed $0.01 \mathrm{~mm}$ and refinement size of 0.005 $\mathrm{mm}$. It is worth mentioning that the approach with refined mesh is computationally more efficient.

Mesh convergence has been found with a refined mesh of $0.001 \mathrm{~mm}$. Figure 3 (a) shows that the local stress overlap using refined seeds of 0.001 and $0.0002 \mathrm{~mm}$. In addition, the shear modulus $G_{a}$ has also been calculated in the convergence study. When the element size decreases, the value $G_{a}$ decreases and approaches the constant value of $3610 \mathrm{MPa}$, see Figure 3 (b). The difference between the predicted values of $G_{a}$ using seeds of 0.001 and $0.0002 \mathrm{~mm}$ is only $2 \mathrm{MPa}$.

The same conclusions have been obtained in a convergence study using GFRP1 (Set-2). Therefore, the global seed used in almost all simulations is $0.01 \mathrm{~mm}$ with refinement elements around the tip and close to the free surfaces with a size of $0.001 \mathrm{~mm}$. It is important to mention that, for the RUCs which represent higher crack densities, the global size has been always decreased until having more than 20 elements along the $x$-direction. 


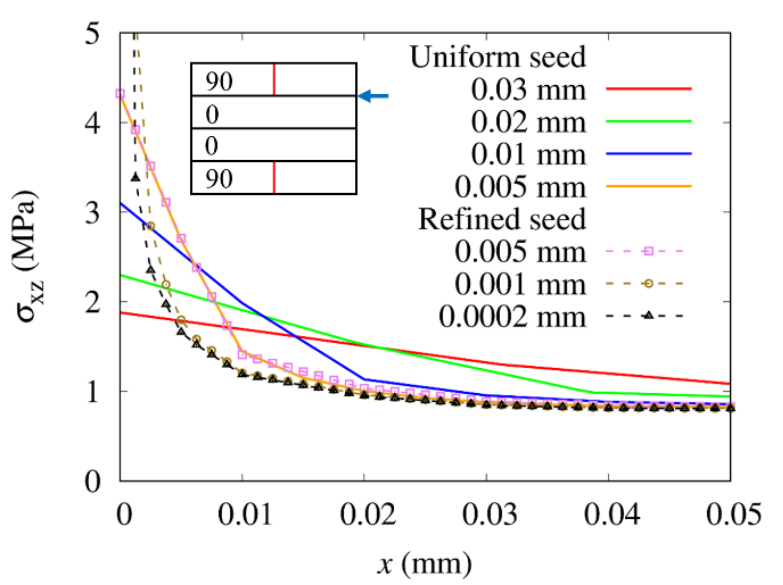

(a)

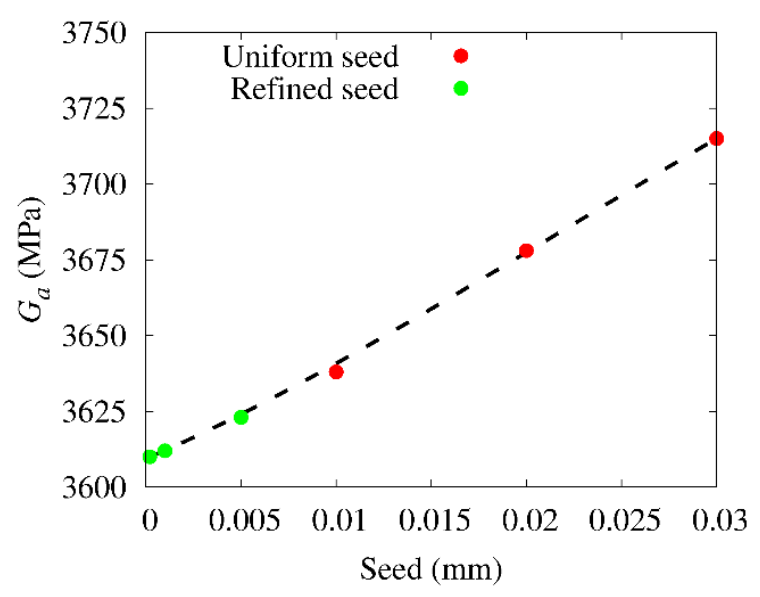

(b)

Figure 3. (a) Local shear stress $\sigma_{x z}$ along the interface 90/0 for different element sizes and refinements at the crack tip. It is worth mentioning that all the refined results agree far from the crack tip when $x>0.1 \mathrm{~mm}$. (b) Shear modulus $G_{a}$ as a function of the element size for uniform and refined seeds. [90/0]s laminate with crack density 2/mm made of CFRP1 (Set-1) under shear load $\tilde{\sigma}_{x z}=1 \mathrm{MPa}\left(\mathrm{N}_{x z}=0.576 \mathrm{~N} / \mathrm{mm}\right)$ using relaxed $3 \mathrm{D}$ PBCs.

The stress singularity at the crack tip increases when the size of the elements decreases, because homogenized linear elastic ply properties have been assumed in this study. Note that the size of the refined elements is smaller than the diameter of the reinforcement fibers. Therefore, local effects due to the microstructure of each ply are not considered in the local stresses calculated with this mesoscale model.

\subsubsection{Local stress fields under in-plane axial loads. Comparison with analytical and numerical models.}

A comparison with published numerical data has been performed to validate the predicted local stress distributions. A symmetric [0/90/-45/45] laminate with crack density $0.2 / \mathrm{mm}$ in $90^{\circ}$ plies made of GFRP2 (Set-3) is considered under normal in-plane load, $\widetilde{\sigma}_{\mathrm{xx}}=50 \mathrm{MPa}\left(\mathrm{N}_{\mathrm{xx}}=200 \mathrm{~N} / \mathrm{mm}\right)$. This laminate has been already studied by Tong et al. [22] and McCartney [17] using Finite Elements and the Generalized Plane Strain approach (the NPL model), respectively. In this section, the local stresses have been calculated via FE with relaxed 3D PBCs and the Variational Approach (VA) described in [33]. Figure 4 (a) shows the local stress $\sigma_{x x}$ along the thickness of the laminate at the plane that contains the cracks. There is a good correlation between the published numerical results and the new results using the FE model with relaxed 3D PBCs and the VA. The variational approach is applied using a ply refinement technique to ensure having accurate converged results. To do so, each ply in the laminate is first divided into five ply elements of equal thicknesses. Further, ply elements adjacent to interfaces are subdivided in half, two times. All of them show that $\sigma_{x x}$ reaches a singularity at the interfaces 90/45 and 0/90. In addition, the local stress $\sigma_{x z}$ along the interface $0 / 90$ has been represented in Figure 4 (b). Again, the results of all methods match notably. It is important to remark that the hypothesis of Generalized Plane Strain used in the previous studies is compatible with the imposed PBCs in transverse direction for this symmetric laminate. 


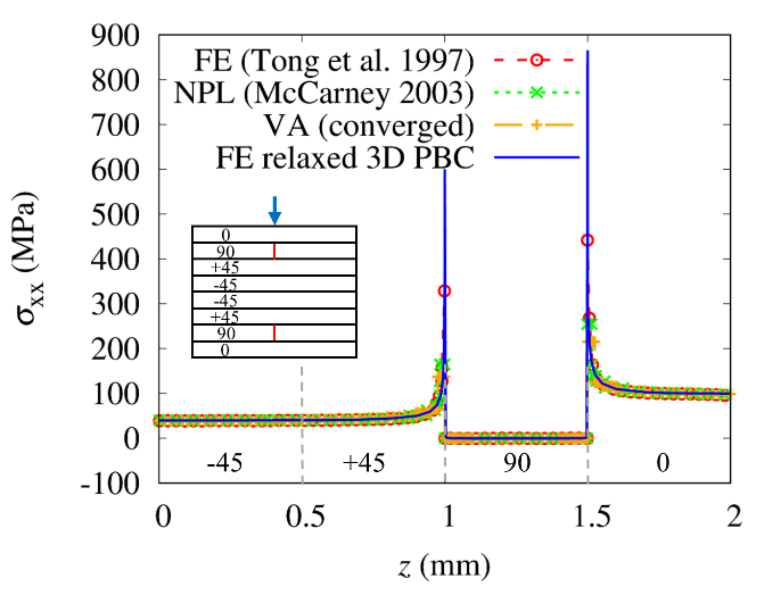

(a)

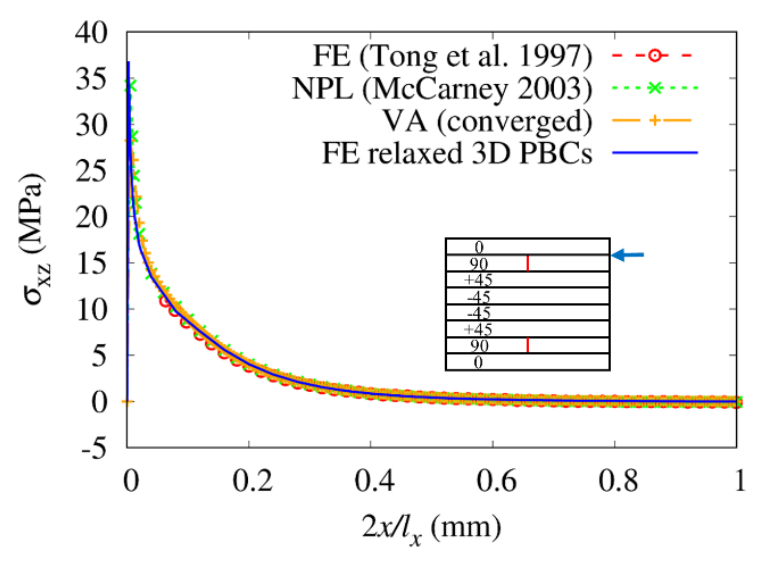

(b)

Figure 4. (a) Axial stress $\sigma_{x x}$ through the thickness at the plane which contains the crack, (b) shear stress $\sigma_{x z}$ at the interface 0/90 of the 90 ply. [0/90/-45/45]s laminate with crack density 0.2/mm made of GFRP2 (Set-3) under axial load with average stress $\tilde{\sigma}_{x x}=50 \mathrm{MPa}\left(\mathrm{N}_{x x}=200 \mathrm{~N} / \mathrm{mm}\right)$

\subsubsection{Local stress field due to in-plane shear load and uniform out-of-plane normal load. Comparison between FE and VA methods.}

Although there are results for local stresses in symmetric laminates under in-plane shear load [12], they have never been reported with high accuracy around the crack tips. In this section, the local stress along the thickness and along the interface $30 / 90$ of the $\left[30 / 90_{8} / 30_{0.5}\right]_{s}$ laminate have been reported. The $\left[30 / 90_{8} / 30_{0.5}\right]_{\mathrm{s}}$ laminate made of GFRP1 (Set-2) with a crack density of $1 / \mathrm{mm}$ has been loaded under in-plane shear $\widetilde{\sigma}_{\mathrm{xy}}=30 \mathrm{MPa}\left(N_{x y}=82.08 \mathrm{~N} / \mathrm{mm}\right)$ and out-of-plane normal $\widetilde{\sigma}_{\mathrm{zz}}=10 \mathrm{MPa}$ $\left(N_{z z}=27.36 \mathrm{~N} / \mathrm{mm}\right)$. The out-of-plane normal stress has been introduced as a uniform stress for both methods, FE with relaxed 3D PBCs and VA. This load condition can be representative of a composite pressure vessel under torsion.

Figure 5 (a) shows the normal stress $\sigma_{z z}$ and shear stresses $\sigma_{x y}$ and $\sigma_{y z}$ across the thickness at $x=0$, which is the plane that contains the cracks. As expected, $\sigma_{x y}$ is zero at the crack surfaces and there are singularities at the crack tips. In Figure 5 (b), the same stresses, $\sigma_{z z}, \sigma_{x z}$ and $\sigma_{y z}$, are represented along the $30 / 90$ interface of the $90^{\circ}$ ply. $\sigma_{z z}$ is symmetric with respect to the crack plane $(x=0)$ while $\sigma_{x z}$ and $\sigma_{y z}$ are anti-symmetric. There is perfect accordance between the FE and VA methods in Figure 5, where the FE results are represented by solid lines while VA results by dots. 


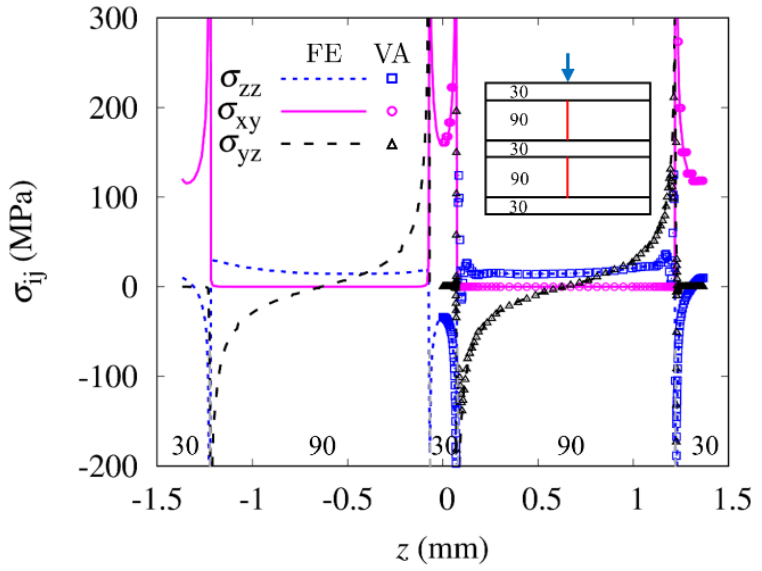

(a)

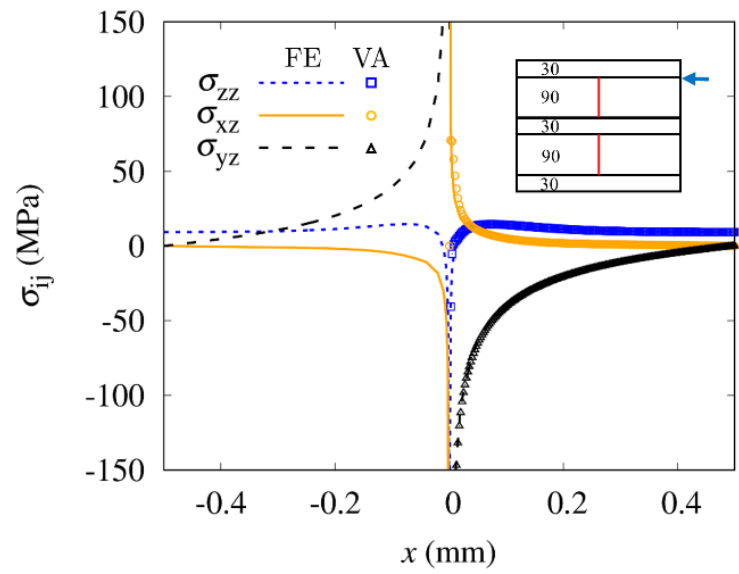

(b)

Figure 5 (a) Local axial and shear stresses through the thickness at the plane which contains the crack, (b) local stresses at the interface $30 / 90$ of the $90^{\circ}$ ply. [30/908/30 0.5$]_{s}$ laminate with crack density $1 / \mathrm{mm}$ made of GFRPI (Set-2) under shear and axial loads, $\tilde{\sigma}_{x y}=30 \mathrm{MPa}$ and $\tilde{\sigma}_{z z}=10 \mathrm{MPa}\left(N_{x y}=82.08 \mathrm{~N} / \mathrm{mm}\right.$ and $N_{z z}=27.36 \mathrm{~N} / \mathrm{mm}$ ).

\subsubsection{Local stress field due to out-of-plane shear load $\widetilde{\sigma}_{x z}$}

In this section, the capabilities of the FE model with relaxed 3D PBCs are shown when the out-ofplane shear load $N_{x z}$ is applied to the RUC. The [90/0]s laminate with crack density $2 / \mathrm{mm}$ made of GFRP1 (Set-2) is considered in this example. The out-of-plane shear load has been introduced as concentrated forces in the control points.

Figure 6 (a) shows the important stresses along the plane which contains the crack when the $[90 / 0]_{\mathrm{s}}$ laminate is loaded with $\tilde{\sigma}_{x z}=1.736 \mathrm{MPa}\left(N_{x z}=1 \mathrm{~N} / \mathrm{mm}\right)$. Although the shear stress $\sigma_{x z}$ is zero at the crack surfaces, the transverse stress $\sigma_{y y}$ and normal stress $\sigma_{z z}$ stresses are notable at the crack planes. In Figure 6 (b), the results along the $90 / 0$ interface in the $90^{\circ}$ ply show that the normal stresses are anti-symmetric with respect to the crack plane and they are only important at the singularities.

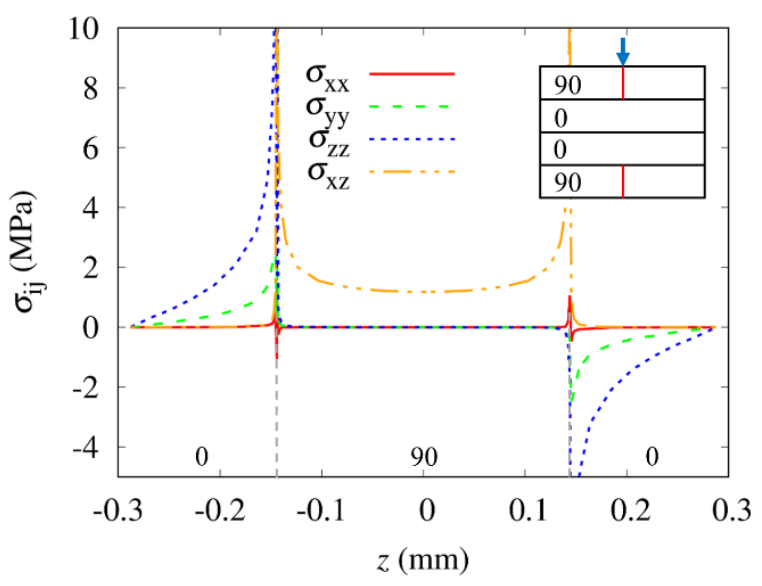

(a)

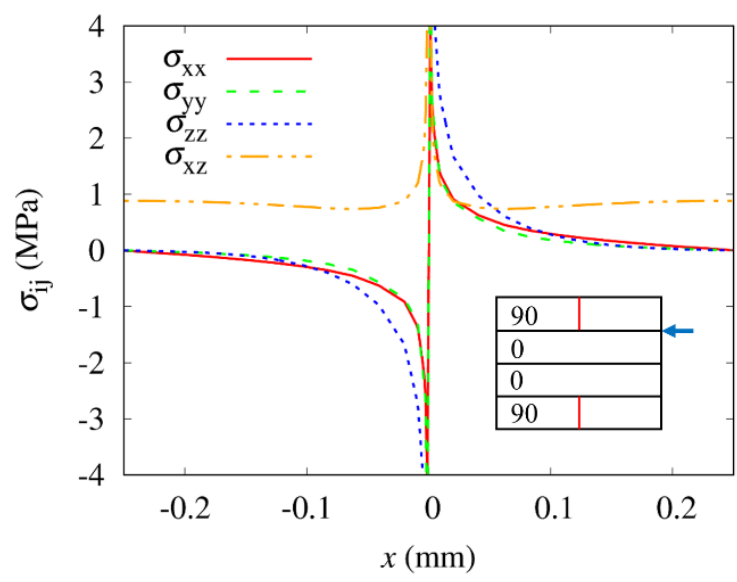

(b)

Figure 6. (a) Stresses through the thickness at the crack plane, (b) stresses at the interface $90 / 0$ in the $90^{\circ}$ ply. $[90 / 0]_{s}$ laminate with crack density $2 / \mathrm{mm}$ made of GFRP1 (Set-2) under shear load $\tilde{\sigma}_{x z}=1.736 \mathrm{MPa}\left(N_{x z}=1\right.$ $\mathrm{N} / \mathrm{mm}$ ).

The normal and transverse displacements $u_{x}$ and $u_{z}$ along the plane that contains the cracks are reported in Figure 7 (a) for the same load condition $\tilde{\sigma}_{x z}=1.736 \mathrm{MPa}\left(N_{x z}=1 \mathrm{~N} / \mathrm{mm}\right)$. The 
displacements of the nodes for the left and right surfaces of the crack are distinguished. While $u_{x}$ is the same for both crack surfaces, $u_{z}$ shows the slipping behavior at the crack surfaces. In Figure 7 (b), the normal and transverse displacements $u_{x}$ and $u_{z}$, and the shear stress $\sigma_{x z}$ are shown along the top surface. When relaxed 3D PBCs are applied, the stress $\sigma_{x z}$ is not uniform between cracks as expected. The authors are aware about the limitation of representing stresses at surfaces using FE analysis based on displacement method. Therefore, a refined mesh close to the free surfaces has been considered in the current study. The transverse displacement $u_{x}$ of the nodes at the top surface is constant, while the normal displacement $u_{z}$ has a discontinuity at the crack plane.

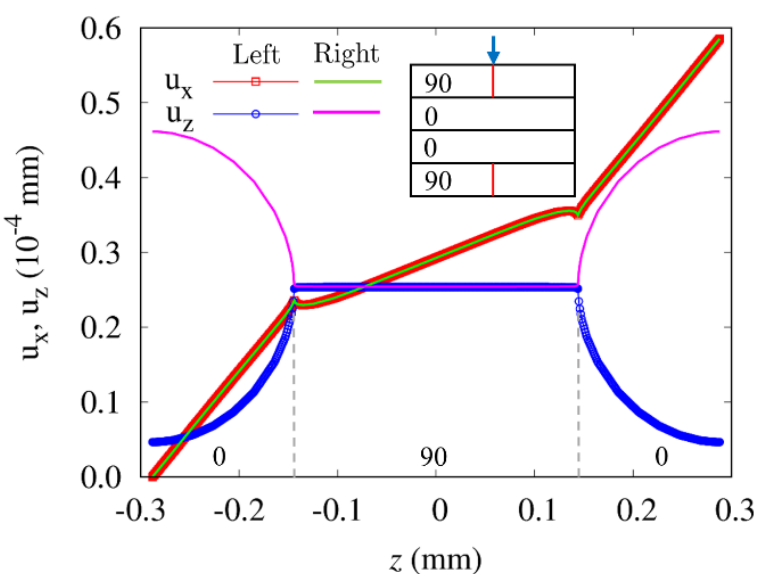

(a)

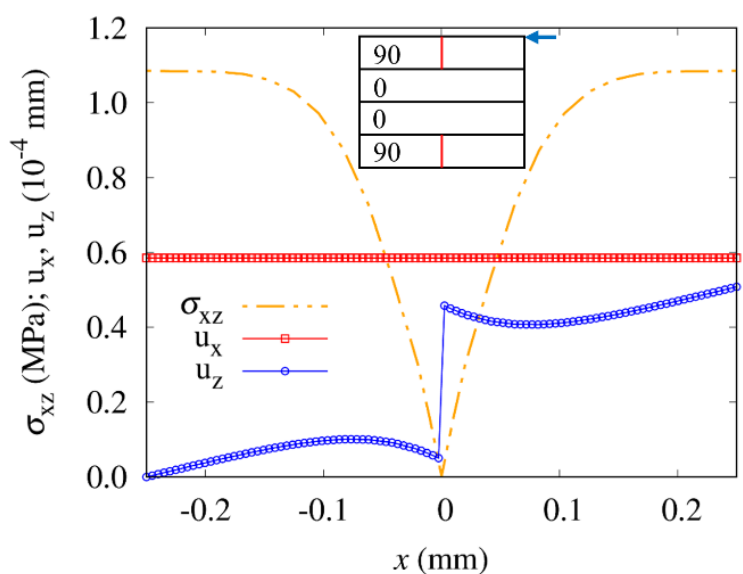

(b)

Figure 7. (a) Normal and transverse displacements $u_{x}$ and $u_{z}$ along the plane which contains the crack surfaces where the left and right faces are distinguished. (b) Normal and transverse displacements $u_{x}$ and $u_{z}$, and shear stress $\sigma_{x z}$ at the top surface. [90/0] laminate with crack density 2/mm made of GFRP1 (Set-2) under shear load $\tilde{\sigma}_{x z}=1.736 \mathrm{MPa}\left(\mathrm{N}_{x z}=1 \mathrm{~N} / \mathrm{mm}\right)$ using relaxed $3 \mathrm{D} P B C \mathrm{~s}$.

\subsubsection{Local stress field of angle-ply laminates $[90 / \theta]$ with and without contact between crack planes}

330 Previous results have been calculated without considering the possible contact between the crack surfaces under certain load conditions. This assumption is usually proposed within models that represent the cracked laminates. However, when angle-ply laminates are studied, the effects due to the contact between the crack surfaces must be analyzed. For the sake of clarity, the [90/45]s laminate with crack density $2 / \mathrm{mm}$ in $90^{\circ}$ plies made of GFRP1 (Set-2) has been studied under in-plane shear load $\tilde{\sigma}_{x y}=1.736 \mathrm{MPa}\left(N_{x y}=1 \mathrm{~N} / \mathrm{mm}\right)$. Contact between the crack surfaces is considered with friction. Therefore, a hard contact model with isotropic friction based on the penalty method has been added at the crack surfaces using a default friction coefficient of 0.4 . Figure 8 (a) shows the contact pressure at the crack surfaces, which has a singularity at the crack tip. It is noteworthy that, away from the crack tip, the contact pressure has a minimum value of $0.6 \mathrm{MPa}$ which is $1 / 3$ of the imposed load.

To evaluate the local effect of the contact, the stresses along the $90 / 45$ interface in the $90^{\circ}$ ply have been calculated for both the models with and without contact. It can be seen in Figure 8 (b) that the $\sigma_{x x}, \sigma_{x y}, \sigma_{z x}$ and $\sigma_{y z}$ stresses show slightly different distributions when the contact between crack surfaces is considered. When contact is considered, the stress singularities are less pronounced affecting a smaller area close to the crack tip and, therefore, the homogenized stresses are reduced. These qualitative results with contact lead to a non-conservative solution. In addition, the same load 
case with frictionless contact has been studied. The local stresses $\sigma_{x x}$ and $\sigma_{z x}$ remain the same with and without friction, while the local stresses $\sigma_{x y}$ and $\sigma_{y z}$ are different when the friction coefficient is changed. It is noteworthy that calculated local stresses are under the hypothesis of homogenized linear elastic ply properties.

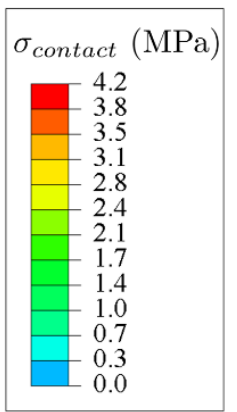

(a)

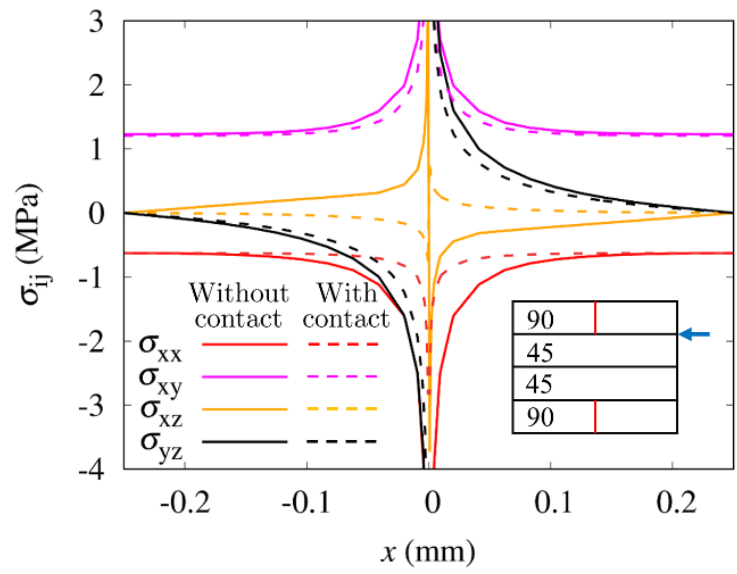

(b)

Figure 8. (a) Contact pressure at the crack surfaces of the deformed RUC with a scale factor of 750. (b) Stresses at the interface 90/45 in the $90^{\circ}$ ply when contact between crack planes is considered or not. [90/45]s laminate with crack density $2 / \mathrm{mm}$ made of GFRPl (Set-2) under shear load $\tilde{\sigma}_{x y}=1.736 \mathrm{MPa}\left(\mathrm{N}_{x y}=1 \mathrm{~N} / \mathrm{mm}\right)$.

The contact between crack surfaces does not notably affect the results under other loads such as axial loads and out-of-plane shear loads. The relevant load cases for a selected laminate can be identified looking at the homogenized behavior of the laminate when it is retrieved with and without contact. For the [90/45] laminate, the homogenized properties $G_{A}$ and $\lambda_{x}$ show differences larger than $4 \%$ when contact between crack planes is considered. This indicates that there are only notable differences in the local stresses under in-plane shear $N_{x y}$. It is noted that when contact with friction between crack surfaces is used, the laminate stiffness depends on the applied stress level. However, for the case without friction, the stiffness terms remain constant using a perturbation stress level below $1 \mathrm{MPa}$ because the same portion of cracked surfaces is in contact.

As a conclusion, the contact between crack surfaces must be only considered in some specific cases, where the closing of the crack modifies the local stresses and the homogenized properties. Conservatively, the cracks can always be considered open, and therefore, the simulations can be performed without considering the contact leading to larger stiffness reductions. In the following sections, the simulations are done without considering contact unless the opposite is mentioned.

\subsection{Stiffness reduction}

In this section, degradation of the effective laminate thermo-elastic properties due to uniform ply cracking is reported. First, the FE model has been verified and validated with published data. Then, degraded effective properties including the ones related to out-of-plane loads are reported. Finally, the results for cross-ply and angle-ply laminates are discussed. 


\subsubsection{In-plane stiffness reduction. Comparison with analytical, numerical and experimental data.}

Figure 9 (a) and (b) show the reduction of the normalized axial modulus $\left(E_{A}\right)$ as a function of the crack density, $\rho$, for two different laminates: (i) the $\left[0 / 90_{8} / 0_{0.5}\right]_{s}$ laminate made of GFRP1 (Set-2) containing cracks in the $90^{\circ}$ plies; (ii) and the [0/45] laminate made of GFRP5 (Set-6) containing cracks in the $45^{0}$ plies. First, the FE model with relaxed 3D PBCs has been verified with the analytical model based on VA and published numerical results. Then, the results are validated versus experimental data.

The verification of the FE model with relaxed 3D PBCs has been done comparing the results with converged results using the VA. Figure 9 (a) shows good agreement between the results of the proposed FE model and the converged results using VA [33]. In addition, the calculated normalized $E_{A}$ have also been compared with published FE results $[6,13]$ which are not included in Figure 9 (a) for the sake of clearness. The differences are lower than $1.5 \%$ when published data is compared to the current FE implementation with relaxed 3D PBCs.

Figure 9 (a) also includes the results of the FE model with 3D PBCs formulation (FE 3D PBCs) in which the periodicity of the normal displacement between top and bottom is incorporated. As expected, the FE model with 3D PBCs gives stiffer values of the axial modulus $\left(E_{A}\right)$ due to the restriction of the displacements between the top and bottom surfaces. As a conclusion, relaxed 3D $\mathrm{PBCs}$ are needed to retrieve correctly the in-plane behavior of the laminate while out-of-plane shear loads can be applied using only one implementation of PBCs.

It is observed that the numerical results follow the trend of the experimental ones [26], but they overestimate the stiffness reduction, see Figure 9 (a). These discrepancies were studied by Barulich et al. [6] who explained that these differences are due to the non-uniform distribution of the transverse cracks along the plies. This explanation is in agreement with other studies [23] and also with the results presented in section 4.2.

Figure 9 (b) shows the result for the $[0 / 45]_{s}$ laminate with cracks in the $45^{\circ}$ plies. Therefore, FE calculations with the current model have been done for a [45/90]s laminate with cracks in the $90^{\circ}$ plies. Then, the stiffness matrix has been rotated -45 degrees to get the material properties of the initial laminate [0/45]s. There is good agreement between the FE relaxed 3D PBCs and VA. The numerical results are also in good agreement with the experimental data, although it is observed in the experiments that the cracks are non-uniform. The differences between experiments and numerical results at high crack density are commented by Katerelos et al. [34] guessing a non-linear matrix behavior. 


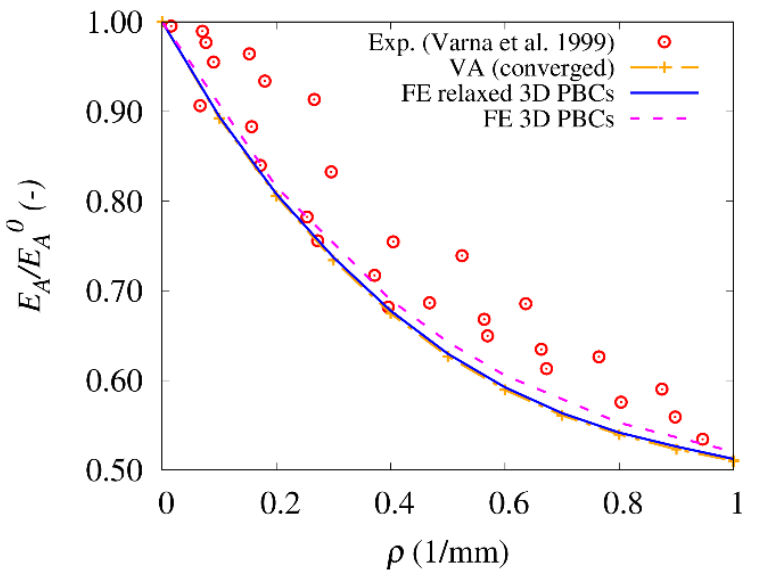

(a)

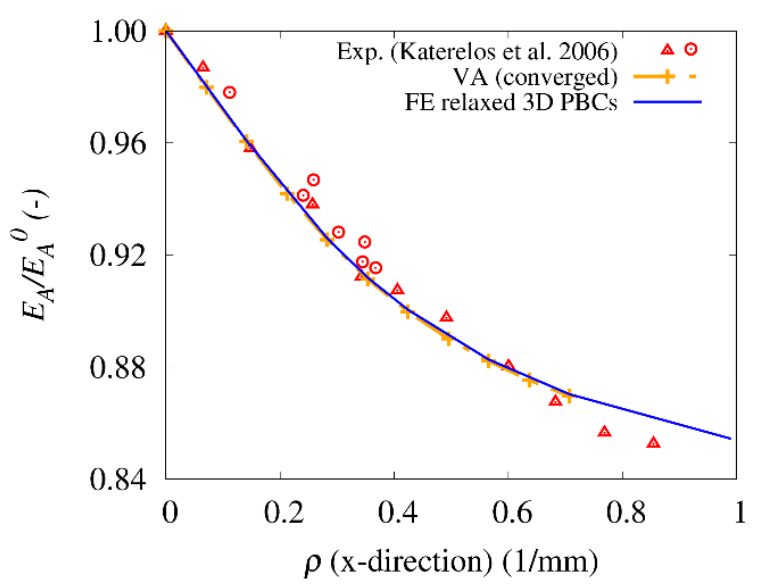

(b)

Figure 9. (a) Normalized axial stiffness as a function of the crack density $\rho$ for the $\left[0 / 90_{8} / 0_{0.5}\right]_{s}$ laminate made of GFRPI (Set-2), cracks in the $90^{\circ}$ plies. (b) Normalized axial stiffness as a function of the crack density $\rho(x-$ direction) for the [0/45]s laminate made of GFRP5 (Set-6), cracks in the $45^{\circ}$ plies.

\subsubsection{Out-of-plane stiffness reduction. Comparison with numerical data.}

410 To the best knowledge of the authors, there are no experimental data available to make a verification of the numerical models when out-of-plane shear stiffness terms are concerned. The current FE model with relaxed 3D PBCs implementation has been verified with numerical results based on a FE analysis [5]. The main difference is that the published FE analysis uses a 3D formulation without relaxing the condition at the free surfaces, top and bottom. The published results in [5] are performed for a [0/90/45/-45]s laminate made of GFRP3 (Set-4) with cracks in the $45^{\circ}$ plies. Therefore, FE calculations have been performed for a [45/-45/90/0 $]_{\mathrm{s}}$ laminate with cracks in the $90^{\circ}$ plies. Then, the stiffness matrix has been rotated -45 degrees to get the effective properties of the initial laminate $[0 / 90 / 45 /-45]_{s}$.

Figure 10 (a) shows the normalized in-plane and out-of-plane effective engineering properties, $E_{A}, E_{T}$, $G_{A}, G_{t}, G_{a}, v_{t}$ and $v_{a}$ for a [0/90/45/-45]s laminate made of GFRP3 (Set-4) with symmetric cracks in the $45^{\circ}$ plies. The normalized properties are presented as a function of the normalized crack density $\left(45^{\circ} \mathrm{ply}\right)$ with the ply thickness $(0.2 \mathrm{~mm})$. The published properties calculated via FE [5] and the current FE simulation with relaxed 3D PBCs are in good agreement. Note that the published data is only available for normalized crack densities from 0 to 2 (corresponding to crack densities from 0 to $10 / \mathrm{mm})$. It is noteworthy that, in the [0/90/45/-45]s laminate, $E_{A}=E_{T}, G_{t}=G_{a}$ and $v_{t}=v_{a}$ because the cracks are in the $45^{\circ}$ ply. It has been checked that $G_{t}$ remains constant in cross-ply laminates as in the case of the $[90 / 0]_{\mathrm{s}}$ laminate with cracks in the $90^{\circ}$ plies, see section 3.1. In the latter laminate, the cracks do not affect the shear load $N_{y z}$ and, therefore, the behavior is as an intact laminate under this load condition. Finally, to provide a data set for future benchmarks the effective engineering properties as a function of crack density have been reported in a large range of normalized crack densities from 0 to 100. It is remarkable that the out-of-plane properties degrade more than the inplane properties and approach to zero for large crack densities. In addition, the normalized out-ofplane Poisson's ratio $v_{t}=v_{a}$ degrades up to $20 \%$ of its original value when the crack density increases. 
Figure 10 (b) also shows good agreement between published and current results for the coupling ratios $\left(\lambda_{x}, \lambda_{y}, \lambda_{z}\right.$, and $\left.\lambda_{S}\right)$. Note that $\lambda_{z}^{*}=\lambda_{z} S_{11} / S_{33}$ is the coupling coefficient used in [5]. The studied range of coupling coefficients has been extended until a normalized crack density of 100 to provide more data for future benchmarks. The in-plane and out-of-plane coupling coefficients decrease from zero when the crack density increases. As expected, the shear components are completely coupled $\left(\lambda_{S}=-1\right)$ at high crack density because the laminate becomes unbalanced following the ply-discount solution.

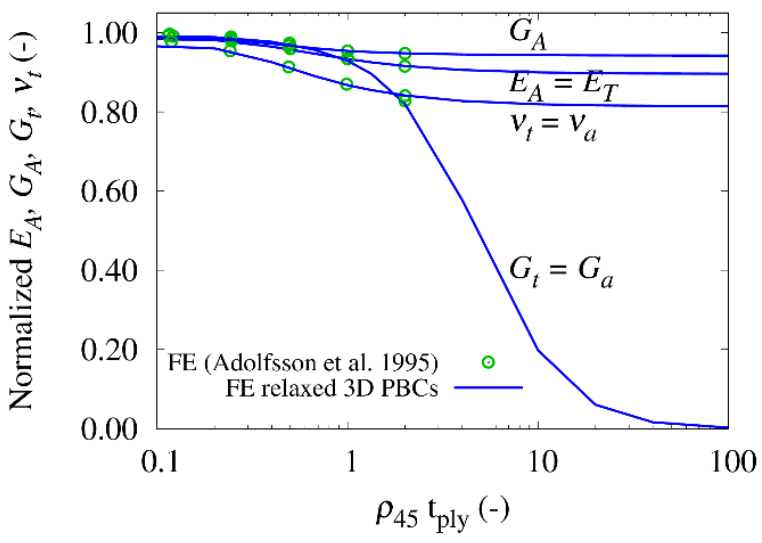

(a)

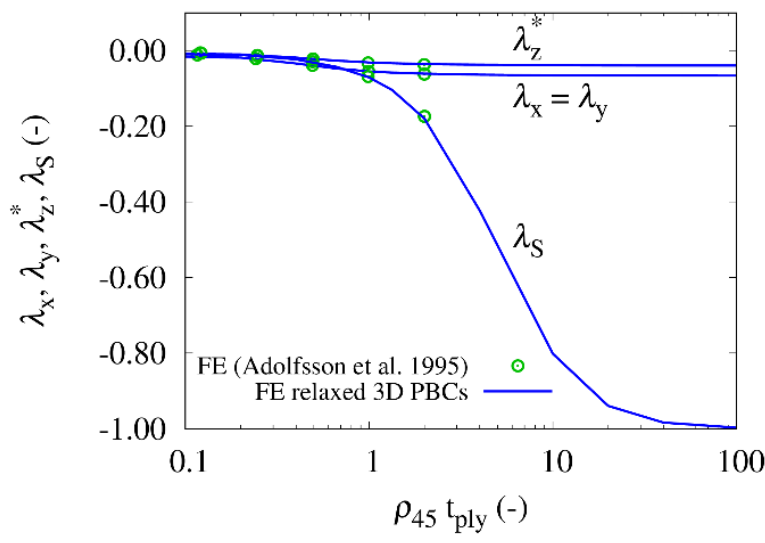

(b)

Figure 10. (a) Normalized axial, shear stiffness and Poisson's ratio, and (b) coupling coefficients ratios as a function of the normalized crack density with the ply thickness, $\rho_{45} t_{p l y}$. [0/90/45/-45]s laminate made of GFRP3 with symmetric cracks in the $45^{\circ}$ ply. Note that $\lambda_{z}^{*}=\lambda_{z} S_{11} / S_{33}$.

445 As a conclusion, the reduction of the out-of-plane properties should certainly be taken into account.

\subsubsection{Reduction of the effective engineering properties of cross-ply and angle-ply laminates.}

The effective engineering properties of different cracked laminates $[90 / \theta]_{\mathrm{s}}$ and $\left[\theta / 90_{8} / \theta_{0.5}\right]_{\mathrm{s}}$ with crack density $2 / \mathrm{mm}$ in $90^{\circ}$ plies have been calculated applying relaxed 3D PBCs. Table 2 and Table 3 show the normalized effective engineering properties for laminates made of GFRP1 (Set-2) and CRFP1

450 (Set-1), respectively. The normalization of the properties was done using the absolute value of the effective engineering properties obtained from the intact laminate (without cracks). The intact values $\lambda_{z}$ and $\alpha_{S}$ are negative for all the studied laminates and materials. Also, the intact value of $\alpha_{T}$ is negative for $\left[45 / 90_{8} / 45_{0.5}\right]_{\mathrm{s}}$ and $\left[60 / 90_{8} / 60_{0.5}\right]_{\mathrm{s}}$ laminates made of CFRP1 (Set-1). For laminates with thicker $90^{\circ}$ plies, e.g. $\left[\theta / 90_{8} / \theta_{0.5}\right]_{\mathrm{s}}$, the reduction of the properties is more pronounced. The laminates made of CFRP1 (Set-1) show more influence in the thermal expansion coefficients.

Table 2. Normalized engineering properties with respect to the absolute intact properties for different laminates made of GFRP1 (Set-2) with 2/mm crack density. Note that the intact properties $\lambda_{z}$ and $\alpha_{S}$ are negative. Relaxed 3D PBCs have been used.

\begin{tabular}{ccccccccc}
\hline & {$[90 / 0]_{\mathrm{s}}$} & {$[90 / 30]_{\mathrm{s}}$} & {$[90 / 45]_{\mathrm{s}}$} & {$[90 / 60]_{\mathrm{s}}$} & {$\left[0 / 90_{8} / 0_{0.5}\right]_{\mathrm{s}}$} & {$\left[30 / 90_{8} / 30_{0.5}\right]_{\mathrm{s}}$} & {$\left[45 / 90_{8} / 45_{0.5}\right]_{\mathrm{s}}$} & {$\left[60 / 90_{8} / 60_{0.5}\right]_{\mathrm{s}}$} \\
\hline$E_{A}$ & 0.86 & 0.66 & 0.71 & 0.68 & 0.46 & 0.33 & 0.28 & 0.25 \\
$E_{T}$ & 1.00 & 0.40 & 1.00 & 0.99 & 1.00 & 1.00 & 1.00 & 0.99 \\
$E_{t}$ & 0.98 & 1.26 & 0.99 & 1.00 & 0.98 & 0.98 & 0.99 & 1.00 \\
$G_{A}$ & 0.80 & 0.75 & 0.82 & 0.84 & 0.31 & 0.35 & 0.38 & 0.39 \\
$G_{t}$ & 1.00 & 1.14 & 1.00 & 1.00 & 1.00 & 1.00 & 1.00 & 1.00 \\
$G_{a}$ & 0.84 & 1.00 & 0.85 & 0.85 & 0.31 & 0.30 & 0.30 & 0.30 \\
\hline
\end{tabular}




\begin{tabular}{lcccccccc}
\hline$v_{A}$ & 0.67 & 1.76 & 0.72 & 0.72 & 0.24 & 0.30 & 0.31 & 0.30 \\
$v_{t}$ & 0.96 & 1.05 & 1.02 & 1.02 & 0.94 & 1.00 & 1.03 & 1.03 \\
$v_{a}$ & 0.67 & 0.70 & 0.66 & 0.65 & 0.23 & 0.23 & 0.22 & 0.22 \\
$\lambda_{x}$ & - & 0.57 & 1.21 & 1.36 & - & 2.60 & 2.58 & 3.15 \\
$\lambda_{y}$ & - & 1.15 & 0.80 & 0.78 & - & 1.03 & 0.61 & 0.59 \\
$\lambda_{z}$ & - & -0.49 & -0.77 & -0.77 & - & -0.58 & -0.55 & -0.58 \\
$\lambda_{S}$ & - & 0.52 & 0.85 & 0.85 & - & 0.30 & 0.30 & 0.30 \\
$\alpha_{A}$ & 0.89 & 1.08 & 0.95 & 0.97 & 0.70 & 0.80 & 0.87 & 0.94 \\
$\alpha_{T}$ & 0.98 & 6.67 & 1.01 & 1.01 & 0.98 & 1.00 & 1.01 & 1.01 \\
$\alpha_{t}$ & 0.97 & 0.57 & 0.99 & 1.00 & 0.96 & 0.98 & 0.99 & 0.99 \\
$\alpha_{S}$ & - & -0.34 & -1.08 & -1.14 & - & -1.77 & -1.97 & -2.33 \\
\hline
\end{tabular}

Table 3. Normalized engineering properties with respect to the absolute intact properties for different laminates of CFRPI (Set-1) with 2/mm crack density. Note that the intact properties $\lambda_{z}$ and $\alpha_{S}$ are negative. Relaxed $3 D$ PBCs have been used.

\begin{tabular}{ccccccccc}
\hline & {$[90 / 0]_{\mathrm{s}}$} & {$[90 / 30]_{\mathrm{s}}$} & {$[90 / 45]_{\mathrm{s}}$} & {$[90 / 60]_{\mathrm{s}}$} & {$\left[0 / 90_{8} / 0_{0.5}\right]_{\mathrm{s}}$} & {$\left[30 / 90_{8} / 30_{0.5}\right]_{\mathrm{s}}$} & {$\left[45 / 90_{8} / 45_{0.5}\right]_{\mathrm{s}}$} & {$\left[60 / 90_{8} / 60_{0.5}\right]_{\mathrm{s}}$} \\
\hline$E_{A}$ & 0.96 & 0.77 & 0.72 & 0.68 & 0.75 & 0.36 & 0.29 & 0.26 \\
$E_{T}$ & 1.00 & 1.00 & 1.00 & 0.99 & 1.00 & 1.00 & 1.00 & 0.98 \\
$E_{t}$ & 0.97 & 0.98 & 0.99 & 1.00 & 0.94 & 0.96 & 0.98 & 0.99 \\
$G_{A}$ & 0.79 & 0.77 & 0.78 & 0.84 & 0.31 & 0.32 & 0.37 & 0.45 \\
$G_{t}$ & 1.00 & 1.00 & 1.00 & 1.00 & 1.00 & 1.00 & 1.00 & 1.00 \\
$G_{a}$ & 0.83 & 0.84 & 0.84 & 0.84 & 0.30 & 0.29 & 0.29 & 0.29 \\
$v_{A}$ & 0.68 & 0.69 & 0.69 & 0.70 & 0.24 & 0.29 & 0.29 & 0.30 \\
$v_{t}$ & 0.93 & 0.99 & 1.05 & 1.07 & 0.86 & 1.00 & 1.12 & 1.14 \\
$v_{a}$ & 0.70 & 0.67 & 0.66 & 0.66 & 0.25 & 0.23 & 0.23 & 0.23 \\
$\lambda_{x}$ & - & 1.08 & 1.08 & 1.14 & - & 1.75 & 1.65 & 1.87 \\
$\lambda_{y}$ & - & 1.00 & 0.78 & 0.74 & - & 1.09 & 0.47 & 0.43 \\
$\lambda_{z}$ & - & -0.72 & -0.71 & -0.72 & - & -0.39 & -0.37 & -0.40 \\
$\lambda_{S}$ & - & 0.84 & 0.84 & 0.84 & - & 0.29 & 0.29 & 0.29 \\
$\alpha_{A}$ & 0.50 & 0.84 & 0.91 & 0.95 & 0.19 & 0.60 & 0.76 & 0.88 \\
$\alpha_{T}$ & 0.87 & 0.99 & 1.17 & 1.62 & 0.50 & 1.03 & 0.86 & -0.01 \\
$\alpha_{t}$ & 0.93 & 0.96 & 0.98 & 0.99 & 0.86 & 0.91 & 0.95 & 0.98 \\
$\alpha_{S}$ & - & -0.93 & -0.98 & -1.05 & - & -1.10 & -1.26 & -1.56 \\
\hline
\end{tabular}

It is worth mentioning that the reduction of some out-of-plane properties is as large as the in-plane ones for all the laminates and materials studied. The shear stiffness $G_{a}$ decreases as much as the inplane shear stiffness $G_{A}$. Also, the Poisson's ratio $v_{a}$ changes as much as $v_{A}$. However, the reduction of through-thickness properties $E_{t}$ and $\alpha_{t}$ are only notable for the cross-ply laminates. In the case of angle-ply laminates with $\theta>0$, the coupling coefficients $\left(\lambda_{x}, \lambda_{y}, \lambda_{z}\right.$, and $\left.\lambda_{S}\right)$ and the shear thermal expansion $\alpha_{S}$ become important.

470 When $\theta$ increases, the axial stiffness $E_{A}$ decreases and the axial thermal expansion coefficient $\alpha_{A}$ increases as expected for both laminates and materials, however, the other engineering properties do not change significantly. Small changes are observed in the shear stiffness $G_{A}$ and the shear thermal expansion coefficient $\alpha_{S}$. It is important to mention that $\alpha_{T}$ shows an important relative change in the case of CFRP1 (Set-1) when $\theta$ increases, see Table 3, but the intact values of these laminates are smaller than the intact values of $\alpha_{A}$. Therefore, the absolute changes of $\alpha_{T}$ are not as relevant as other properties. 
Finally, it is important to mention that contact between the crack planes has not been considered in these simulations and, therefore, conservative values of the effective engineering properties have been reported.

\section{Results for laminate with non-uniform distribution of cracks}

The uniform distribution of cracks studied in the previous section is an idealized crack pattern. In reality, a non-uniform distribution of cracks is expected due to the presence of manufacturing defects. When the crack spacing is non-uniform, the local stress between cracks is different leading to a different stiffness reduction. Different studies which consider the distribution of cracks show that axial stiffness degrades more when the cracks have a uniform distribution.

In order to study in detail a laminate with non-uniform distribution of cracks, a fixed geometry with a random distribution of cracks has been studied. The main goal of this section is to evaluate the local stresses and stiffness reduction under in-plane and out-of-plane loads in the presence of a non-uniform distribution of ply cracks. First, the local stresses in a laminate with non-uniform distribution of cracks are compared with the one containing uniformly spaced ply cracks. Then, all relevant thermoelastic constants of the laminate involving out-of-plane properties with non-uniform distribution of cracks are compared with that of the laminate with uniform cracks.

\subsection{Local stress analysis}

A [45/90 $]_{\mathrm{s}}$ laminate made of GFRP4 (Set-5) with a crack density of $2 / \mathrm{mm}$ has been used in the present study with a random distribution of cracks. Six cracks are located with non-uniform distribution along the $\mathrm{x}$-axis following the case study in reference [23]. Their location is given by $x=$ $0.09,0.3,1.08,1.5,1.62,2.43 \mathrm{~mm}$. First, the local stresses are verified versus the VA results under inplane axial load $N_{x x}$. Then, the local stresses are reported when the out-of-plane shear load $N_{x z}$ is applied.

\subsubsection{Local stress field due to axial load. Benchmark with published VA data.}

The $\left[45 / 90_{2}\right]_{\mathrm{s}}$ laminate is loaded under $N_{x x}=1 \mathrm{~N} / \mathrm{mm}\left(\tilde{\sigma}_{x x}=1.312 \mathrm{MPa}\right)$ using the relaxed 3D PBCs. Figure 11 (a) shows the local stresses $\left(\sigma_{x x}, \sigma_{x y}, \sigma_{x z}\right)$ along the x-direction at the middle plane of the uncracked 45 ply. The positions of the cracks are indicated with the vertical dashed lines. The stresses are in good agreement with the results based on VA reported in Figures 5,6,7 in [23], although there is a $3.5 \%$ difference at the maximum values. The local stress $\sigma_{x x}$ is compared with the same stress at the middle plane when there is a uniform distribution of cracks, see $\sigma_{x x}$ (uniform) in Figure 11 (a). Although the mean stress value is the same for the non-uniform and uniform distribution of cracks, the non-uniform distribution has higher maximum local stresses when two cracks are close. Figure 11 (b) shows the local stresses $\left(\sigma_{x x}, \sigma_{z z}, \sigma_{x z}\right)$ at the interface $45 / 90$ in the $90^{\circ}$

510 plies. The crack positions are revealed by the stress singularities. The local stress $\sigma_{x z}$ changes its value between two cracks from negative to positive being zero at the middle point. 


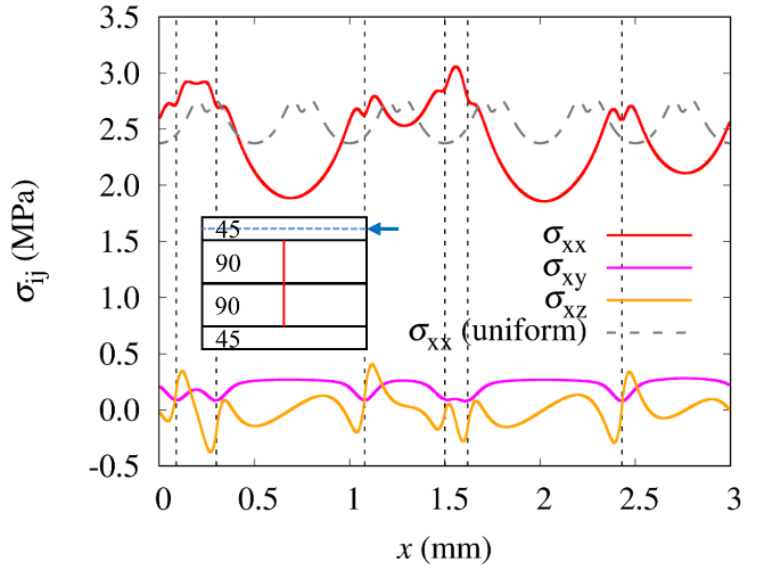

(a)

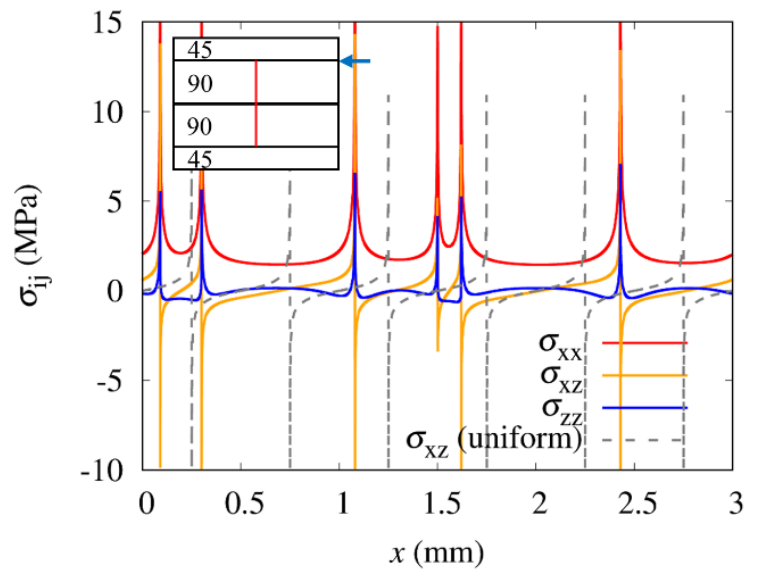

(b)

Figure 11.(a) Stresses at the middle plane of the $45^{\circ} \mathrm{ply}$, and (b) stresses at the interface $45 / 90$ in the $90^{\circ} \mathrm{ply}$. [45/90 $]_{s}$ laminate made of GFRP4 (Set-5) with non-uniform distribution of 6 cracks, crack density 2/mm, under normalized axial load $N_{x x}=1 \mathrm{~N} / \mathrm{mm}\left(\tilde{\sigma}_{x x}=1.312 \mathrm{MPa}\right)$.

515 Figure 12 (a) shows $\sigma_{x x}$ in the deformed transversal plane of the $\left[45 / 90_{2}\right]_{\mathrm{s}}$ laminate. Note that a scale factor of 750 has been used to visualize the displacements. As expected, all the cracks are open due to the axial load, and the stress singularities interfere with each other when the distance between two cracks is short.
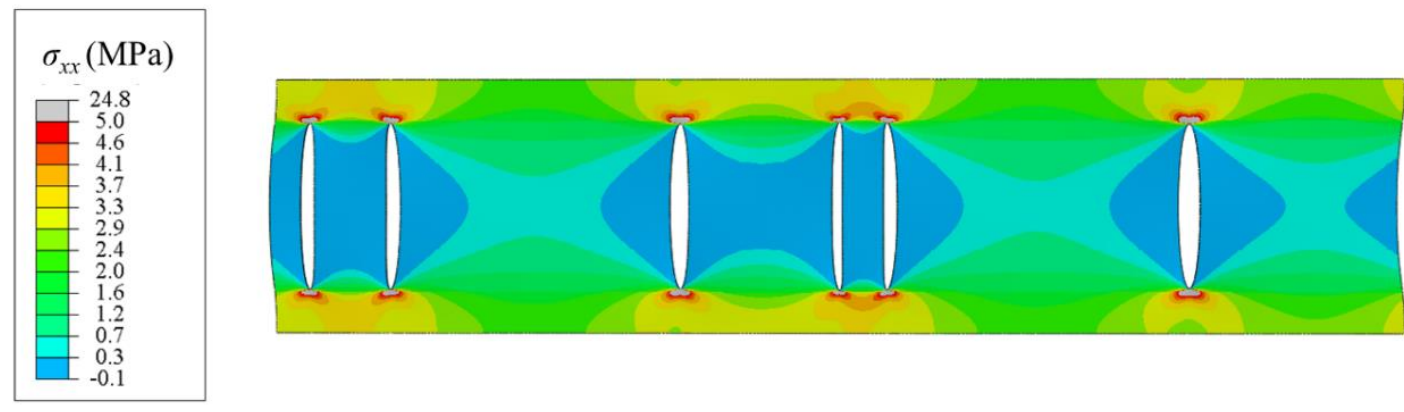

(a)
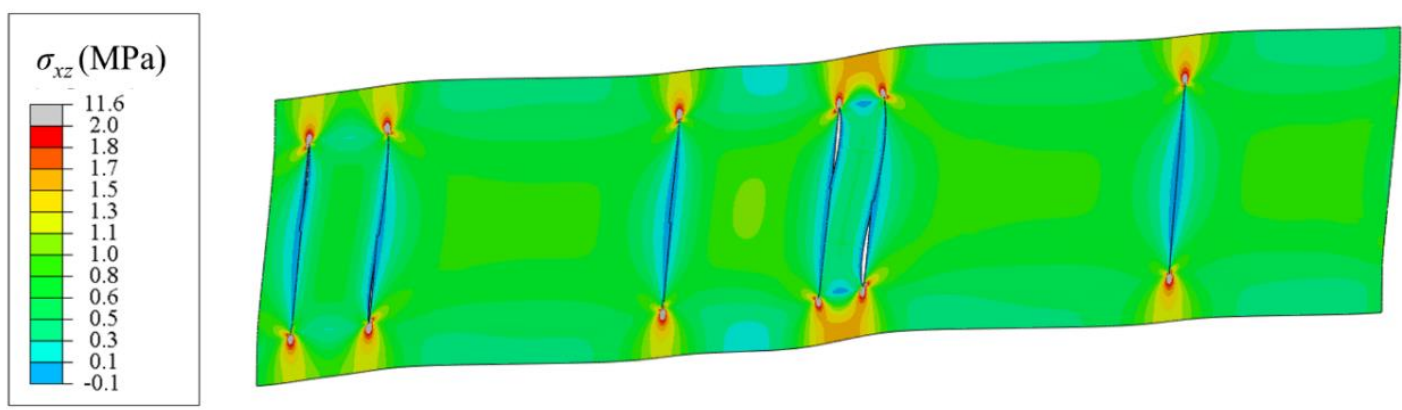

(b)

Figure 12. (a) Stress field $\sigma_{x x}$ under axial load $N_{x x}=1 \mathrm{~N} / \mathrm{mm}$, and (b) stress field $\sigma_{x z}$ under out-of-plane shear load $N_{x z}=1 \mathrm{~N} / \mathrm{mm}\left(\tilde{\sigma}_{x x}=1.312 \mathrm{MPa}\right) .\left[45 / 90_{2}\right]_{s}$ laminate made of GFRP4 with non-uniform distribution of 6 cracks, crack density $2 / \mathrm{mm}$. The deformation has a scale factor of 750 . 


\subsubsection{Local stress field due to an out-of-plane shear load. Future benchmarks.}

In this section, the $\left[45 / 90_{2}\right]_{\mathrm{s}}$ laminate with non-uniform distributed cracks is studied under out-ofplane shear load $N_{x z}=1 \mathrm{~N} / \mathrm{mm}\left(\tilde{\sigma}_{x z}=1.312 \mathrm{MPa}\right)$. The relaxed 3D PBCs formulation allows to impose easily this load condition. Figure 12 (b) shows the stress field $\sigma_{x z}$ in the transversal plane of the $\left[45 / 90_{2}\right]_{\mathrm{s}}$ laminate. All the cracks are mainly closed with a slip displacement between their surfaces. However, when two cracks are in a short distance, the cracks are a little bit open around one of the tips. Note that a scale factor of 750 has been used to represent the displacement in Figure 12 (b). It can be observed again that the stress singularities at the crack tips interfere with each other when the two cracks are in a short distance.

The local stresses $\sigma_{x x}, \sigma_{z z}, \sigma_{x z}$, and $\sigma_{x y}$ have been studied in detail at the middle plane of the 45 ply and at the interface $45 / 90$ in the $90^{\circ}$ ply along the axial direction. Figure 13 (a) shows the local stresses at the middle plane of 45 ply under out-of-plane shear load $N_{x z}=1 \mathrm{~N} / \mathrm{mm}\left(\tilde{\sigma}_{x z}=1.312\right.$ $\mathrm{MPa}$ ). The important stresses at the middle plane of the $45^{\circ}$ ply are $\sigma_{x x}$ and $\sigma_{x z}$, see Figure 13 (a).

The positions of the cracks are located at the maximum values of the out-of-plane shear stress $\sigma_{x z}$. This shear stress $\sigma_{x z}$ with the non-uniform distribution of cracks reaches higher values than the case with a uniform distribution of cracks, see $\sigma_{x z}$ (uniform) in Figure 13 (a).

Figure 13 (b) shows the local stresses along the interface $45 / 90$ in the $90^{\circ}$ ply when the laminate is under out-of-plane shear load $N_{x z}=1 \mathrm{~N} / \mathrm{mm}\left(\tilde{\sigma}_{x z}=1.312 \mathrm{MPa}\right)$. The stress singularities of $\sigma_{z z}$ and $\sigma_{x z}$ at the interface are important because they will trigger delamination under mode I and II. It can be observed that the stress $\sigma_{z z}$ changes from negative to positive values between two cracks, when the laminate is under out-of-plane shear load. It can also be observed that normal out-of-plane perturbation stresses will be zero far from the cracks.

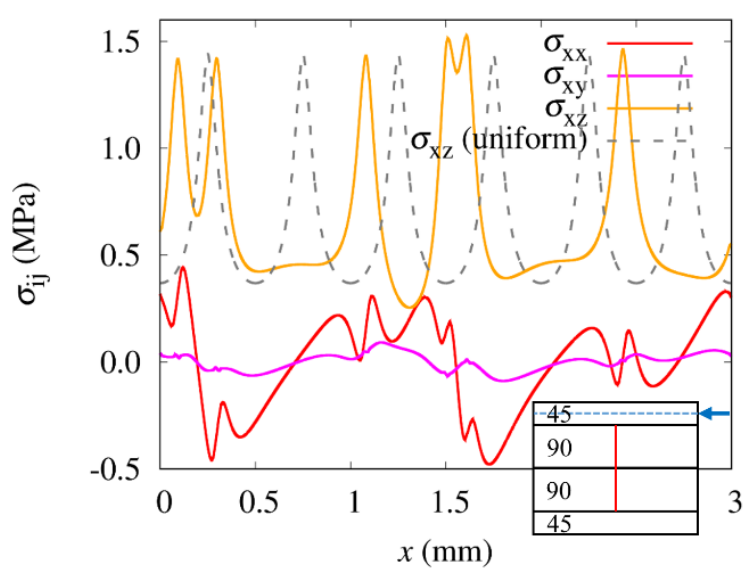

(a)

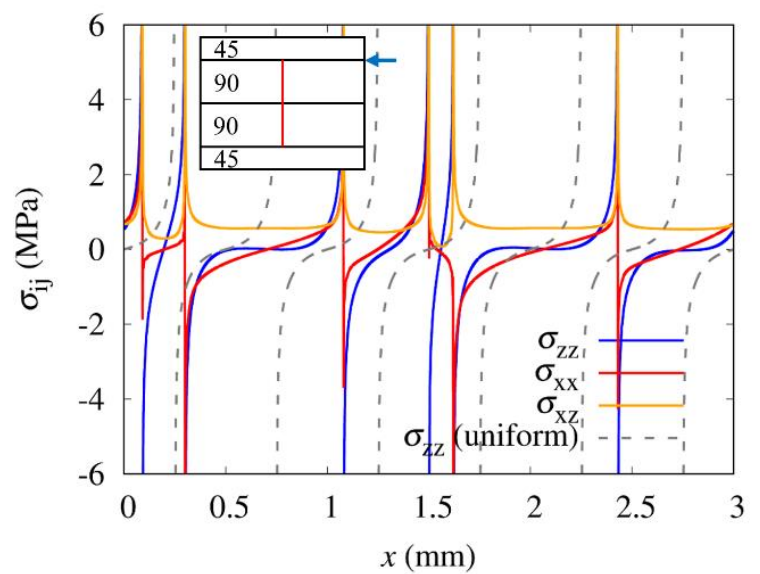

(b)

Figure 13. (a) Stresses at the middle plane of the $45^{\circ}$ ply, and (b) stresses at the interface $45 / 90$ in the $90^{\circ}$ ply.

[45/90 $]_{s}$ laminate made of GFRP4 with non-uniform distribution of 6 cracks, crack density 2/mm, under normalized out-of-plane shear load $N_{x z}=1 \mathrm{~N} / \mathrm{mm}\left(\tilde{\sigma}_{x z}=1.312 \mathrm{MPa}\right)$.

Finally, the same $\left[45 / 90_{2}\right]_{\mathrm{s}}$ laminate under the same out-of-plane shear load has been studied with contact between the crack planes. The results are similar to the case without contact. Therefore, it can be concluded that the contact does not affect this laminate with the studied load case. 


\subsection{Stiffness reduction analysis}

To have a global view of the stiffness reduction, the effective engineering properties for the $\left[45 / 90_{2}\right]_{\mathrm{s}}$ laminate with 6 randomly distributed cracks are compared with the properties for the case with uniform distribution of cracks and same crack density. Table 4 shows the normalized effective engineering properties with respect to the value of an intact laminate. Normalized effective engineering properties using relaxed 3D PBCs have been calculated for two cases, one with uniform and the other one with the non-uniform crack distribution. As expected, the case with uniform crack distribution overestimates the stiffness reduction, then the axial and shear stiffness $\left(E_{A}, E_{T}, G_{A}\right)$ decrease around $4 \%$ with respect to the non-uniform crack distribution case. In addition, the coupling coefficients $\left(\lambda_{x}, \lambda_{y}, \lambda_{z}\right)$ and thermo-expansion coefficients $\left(\alpha_{A}, \alpha_{S}\right)$ are also overestimated with differences of $4 \%$.

Table 4. Normalized effective engineering properties for the uniform and non-uniform symmetric cracked $\left[45 / 90_{2}\right]_{s}$ laminates with crack density $2 / \mathrm{mm}$ using relaxed $3 D \mathrm{PBCs}$.

\begin{tabular}{ccc}
\hline & Uniform & Non-uniform \\
\hline$E_{A}$ & 0.49 & 0.53 \\
$E_{T}$ & 0.99 & 1.00 \\
$E_{t}$ & 0.99 & 0.99 \\
$G_{A}$ & 0.68 & 0.71 \\
$G_{t}$ & 1.00 & 1.00 \\
$G_{a}$ & 0.67 & 0.65 \\
$v_{A}$ & 0.57 & 0.60 \\
$v_{t}$ & 1.03 & 1.03 \\
$v_{a}$ & 0.44 & 0.48 \\
$\lambda_{x}$ & 1.51 & 1.48 \\
$\lambda_{y}$ & 0.62 & 0.64 \\
$\lambda_{z}$ & 0.62 & 0.65 \\
$\lambda_{S}$ & 0.67 & 0.65 \\
$\alpha_{A}$ & 0.90 & 0.91 \\
$\alpha_{T}$ & 1.02 & 1.02 \\
$\alpha_{t}$ & 0.99 & 0.99 \\
$\alpha_{S}$ & 1.26 & 1.23 \\
\hline
\end{tabular}

\section{Conclusions}

A Finite Element (FE) mesoscale model has been developed to study the behavior of a symmetric cracked laminate using a Representative Unit Cell (RUC). Local stresses and effective laminate thermo-elastic constants have been calculated under in-plane and out-of-plane loads. In addition, the contact between crack surfaces and the non-uniform distribution of cracks have been discussed.

Out-of-plane load conditions have been introduced using a relaxed formulation of the threedimensional Periodic Boundary Conditions (relaxed 3D PBCs). This novel formulation allows to combine out-of-plane shear load with in-plane loads keeping free the normal displacement of the top and bottom surfaces. This formulation has been combined with a uniform normal stress applied on the top and bottom surfaces to study the out-of-plane normal behavior. The local stresses at different planes have been compared with analytical and numerical methods showing good agreement. New results under out-of-plane shear load have been reported for future benchmarks. 
To conclude, in order to obtain the in-plane and out-of-plane effective thermo-elastic constants of a symmetric cracked laminate, the novel relaxed 3D PBCs are convenient and efficient. The results of stiffness reduction have been compared with analytical and numerical methods showing good agreement. The approach calculates both the in-plane and out-of-plane effective properties in an accurate way using the same formulation. The calculated in-plane axial stiffness has been compared and validated with experimental data.

The contact between cracks becomes important for specific laminates and load conditions, for example, unbalanced laminates under in-plane shear load. Qualitative local stresses have been compared between the models with and without contact. On the contrary, the hypothesis of open cracks (no contact) can be used when effective material properties are calculated because they give more conservative values.

Finally, the non-uniform distribution of cracks introduces different local stresses between cracks which differ from the uniform case leading to different stiffness reductions. These local effects must be considered together with the stress concentrations due to the local microstructure in the development of new damage models for delamination.

\section{Acknowledgment}

The work leading to this publication has been partially funded by the SBO project "M3Strength", which fits in the MacroModelMat (M3) research program, coordinated by Siemens (Siemens PLM software, Belgium) and funded by SIM (Strategic Initiative Materials in Flanders) and VLAIO (Flemish government agency Flanders Innovation \& Entrepreneurship). M. Hajikazemi acknowledges the financial support of Fonds voor Wetenschappelijk Onderzoek FWO-Vlaanderen (Grant No. G009015N). The work of M. Hajikazemi forms part of the research programme of DPI, project $812 \mathrm{~T} 17$.

\section{Appendix A. Supplementary data}

Supplementary data to this article can be found online at https://doi.org

\section{References}

[1] Talreja R, Varna J. Modeling Damage, Fatigue and Failure of Composite Materials. Elsevier; 2016. doi:10.1016/C2013-0-16521-X.

[2] Ladevèze P. Multiscale Computational Damage Modelling of Laminate Composites. In: Sadowski T, editor. Multiscale Model. Damage Fract. Process. Compos. Mater., Vienna: Springer Vienna; 2005, p. 171-212. doi:10.1007/3-211-38102-3_5.

[3] Crossman FW, Warren WJ, Wang ASD, Law GE. Initiation and Growth of Transverse Cracks and Edge Delamination in Composite Laminates Part 2. Experimental Correlation. J Compos Mater 1980;14:88-108. doi:10.1177/002199838001400107.

[4] Maragoni L, Carraro PA, Quaresimin M. Periodic boundary conditions for FE analyses of a representative volume element for composite laminates with one cracked ply and delaminations. Compos Struct 2018;201:932-41. doi:10.1016/j.compstruct.2018.06.058.

[5] Adolfsson E, Gudmundson P. Matrix crack induced stiffness reductions in [(Om/90n/ $+\theta \mathrm{p} /-$ Өq)s]M composite laminates. Compos Eng 1995;5:107-23. doi:10.1016/0961-9526(95)93983-3. 
[6] Barulich ND, Godoy LA, Dardati PM. Evaluation of cross-ply laminate stiffness with a nonuniform distribution of transverse matrix cracks by means of a computational meso-mechanic model. Compos Struct 2018;185:561-72. doi:10.1016/j.compstruct.2017.11.063.

[7] Dinh TD, Garoz D, Hajikazemi M, Van Paepegem W. Mesoscale analysis of ply-cracked composite laminates under in-plane and flexural thermo-mechanical loading. Compos Sci Technol 2019;175:111-21. doi:10.1016/j.compscitech.2019.03.010.

620 [8] Hajikazemi M, Garoz D, Van Paepegem W. Model to accurately predict out-of-plane shear stiffness reduction in general cracked laminates. Compos Sci Technol 2019;179:88-96. doi:10.1016/j.compscitech.2019.05.009.

[9] Wang ASD, Crossman FW. Initiation and Growth of Transverse Cracks and Edge Delamination in Composite Laminates Part 1. An Energy Method. J Compos Mater 1980;14:71-87. doi:10.1177/002199838001400106.

[10] Nairn JA, Hu S. The initiation and growth of delaminations induced by matrix microcracks in laminated composites. Int J Fract 1992;57:1-24. doi:10.1007/BF00013005.

[11] McCartney LN. Model to predict effects of triaxial loading on ply cracking in general symmetric laminates. Compos Sci Technol 2000;60:2255-79. doi:10.1016/S0266-

[12] Hajikazemi M, Sadr MH. A variational model for stress analysis in cracked laminates with arbitrary symmetric lay-up under general in-plane loading. Int J Solids Struct 2014;51:516-29. doi:10.1016/j.ijsolstr.2013.10.024.

[13] Barbero EJ, Cosso FA, Campo FA. Benchmark solution for degradation of elastic properties due to transverse matrix cracking in laminated composites. Compos Struct 2013;98:242-52. doi:10.1016/j.compstruct.2012.11.009.

[14] Hajikazemi M, Sadr MH. Stiffness reduction of cracked general symmetric laminates using a variational approach. Int J Solids Struct 2014;51:1483-93. doi:10.1016/j.ijsolstr.2013.12.040.

[15] Varna J, Joffe R, Talreja R. A synergistic damage-mechanics analysis of transverse cracking in [ $\pm 0 / 904]$ s laminates. Compos Sci Technol 2001;61:657-65. doi:10.1016/S0266-3538(01)000057.

[16] Yokozeki T, Aoki T. Overall thermoelastic properties of symmetric laminates containing obliquely crossed matrix cracks. Compos Sci Technol 2005;65:1647-54. doi:10.1016/j.compscitech.2005.02.016.

645 [17] McCartney LN. Physically based damage models for laminated composites. Proc Inst Mech Eng Part J Mater Des Appl 2003;217:163-99. doi:10.1177/146442070321700301.

[18] Barbero EJ, Cabrera Barbero J. Analytical Solution for Bending of Laminated Composites with Matrix Cracks. Compos Struct 2016;135:140-55. doi:10.1016/j.compstruct.2015.09.021.

[19] Pupurs A, Varna J, Loukil M, Ben Kahla H, Mattsson D. Effective stiffness concept in bending modeling of laminates with damage in surface 90-layers. Compos Part Appl Sci Manuf 2016;82:244-52. doi:10.1016/j.compositesa.2015.11.012.

[20] Hajikazemi M, Sadr M, Varna J. Analysis of cracked general cross-ply laminates under general bending loads: A variational approach. J Compos Mater 2017;51:3089-109. doi:10.1177/0021998316682364.

[21] Hajikazemi M, Sadr MH, Talreja R. Variational analysis of cracked general cross-ply laminates under bending and biaxial extension. Int J Damage Mech 2015;24:582-624. doi:10.1177/1056789514546010.

[22] Tong J, Guild FJ, Ogin SL, Smith PA. On matrix crack growth in quasi-isotropic laminates-II. Finite element analysis. Compos Sci Technol 1997;57:1537-45. doi:10.1016/S02663538(97)00083-3.

[23] Hajikazemi M, McCartney LN, Van Paepegem W, Sadr MH. Theory of Variational Stress Transfer in General Symmetric Composite Laminates Containing Non-Uniformly Spaced Ply Cracks. Compos Part Appl Sci Manuf 2018. doi:10.1016/j.compositesa.2018.01.021.

[24] Abaqus. User Documentation. Dassault Systemes Simulia Corp, RI, USA; 2017.

[25] Liu S, Nairn JA. The Formation and Propagation of Matrix Microcracks in Cross-Ply Laminates during Static Loading. J Reinf Plast Compos 1992;11:158-78. doi:10.1177/073168449201100204. 
[26] Varna J, Joffe R, Akshantala NV, Talreja R. Damage in composite laminates with off-axis plies. Compos Sci Technol 1999;59:2139-47. doi:10.1016/S0266-3538(99)00070-6.

[27] Gudmundson P, Zang W. An analytic model for thermoelastic properties of composite laminates containing transverse matrix cracks. Int J Solids Struct 1993;30:3211-31. doi:10.1016/00207683(93)90110-S.

[28] Garoz D, Gilabert FA, Sevenois RDB, Spronk SWF, Van Paepegem W. Consistent application of periodic boundary conditions in implicit and explicit finite element simulations of damage in composites. Compos Part B Eng 2019;168:254-66. doi:10.1016/j.compositesb.2018.12.023.

[29] Geers MGD, Kouznetsova VG, Brekelmans WAM. Computational homogenization. In: Gumbsch P, Pippan R, editors. Multiscale Model. Plast. Fract. Means Dislocation Mech. CISM Courses Lect., vol. 522, Wien New York: Springer; 2010, p. 327-94.

[30] Schmitz A, Horst P. A finite element unit-cell method for homogenised mechanical properties of heterogeneous plates. Compos Part Appl Sci Manuf 2014;61:23-32. doi:10.1016/j.compositesa.2014.01.014.

[31] Piezel B, Mercatoris BCN, Trabelsi W, Laiarinandrasana L, Thionnet A, Massart TJ. Bending effect on the risk for delamination at the reinforcement/matrix interface of 3D woven fabric composite using a shell-like RVE. Compos Struct 2012;94:2343-57. doi:10.1016/j.compstruct.2012.03.015.

[32] Espadas-Escalante JJ, van Dijk NP, Isaksson P. A study on the influence of boundary conditions in computational homogenization of periodic structures with application to woven composites. Compos Struct 2017;160:529-37. doi:10.1016/j.compstruct.2016.10.082.

[33] Hajikazemi M, Sadr MH, Hosseini-Toudeshky H, Mohammadi B. Thermo-elastic constants of 690 cracked symmetric laminates: A refined variational approach. Int J Mech Sci 2014;89:47-57. doi:10.1016/j.ijmecsci.2014.08.008.

[34] Katerelos DG, McCartney LN, Galiotis C. Effect of Off - Axis Matrix Cracking on Stiffness of Symmetric Angle-Ply Composite Laminates. Int J Fract 2006;139:529-36. doi:10.1007/s10704006-0100-9. 